\title{
AN EFFICIENT ALGORITHM FOR ACCELERATING THE CONVERGENCE OF OSCILLATORY SERIES, USEFUL FOR COMPUTING THE POLYLOGARITHM AND HURWITZ ZETA FUNCTIONS
}

\author{
LINAS VEPŠTAS
}

\begin{abstract}
This paper sketches a technique for improving the rate of convergence of a general oscillatory sequence, and then applies this series acceleration algorithm to the polylogarithm and the Hurwitz zeta function. As such, it may be taken as an extension of the techniques given by Borwein's "An efficient algorithm for computing the Riemann zeta function" [4 5], to more general series. The algorithm provides a rapid means of evaluating $\operatorname{Li}_{s}(z)$ for general values of complex $s$ and the region of complex $z$ values given by $\left|z^{2} /(z-1)\right|<4$. This region includes the the Hurwitz zeta $\zeta(s, q)$ for general complex $s$ and real $1 / 4 \leq q \leq 3 / 4$. By using the duplication formula and the inversion formula, the range of convergence for the polylogarithm may be extended to the entire complex $z$-plane, and so the algorithms described here allow for the evaluation of the polylogarithm for all complex $s$ and $z$ values. Similarly, the Hurwitz zeta can be extended to the whole real interval $0<q<1$. The speed of the algorithm is significantly superior to a Taylor's series for the evaluation of the Hurwitz zeta; quadratically so when a large number of digits of precision is required. The primary, concrete result of this paper is an algorithm allows the exploration of the Hurwitz zeta in the critical strip, where fast algorithms are otherwise unavailable. A discussion of the monodromy group of the polylogarithm is included.
\end{abstract}

\section{INTRODUCTION}

This note sketches a technique for improving the rate of convergence of a general oscillatory series, and then applies this technique to the computation of the polylogarithm, and, in particular, to the Hurwitz zeta function. It essentially generalizes an algorithm given by Peter Borwein for computing the Riemann zeta function. The principle result is an algorithm that efficiently obtains values of the Hurwitz zeta in the critical strip $s=\sigma+i \tau$ with $0 \leq \sigma \leq 1$.

The Hurwitz zeta function may be defined as

$$
\zeta(s, q)=\sum_{n=0}^{\infty} \frac{1}{(n+q)^{s}}
$$

This series may be directly summed for $\sigma>1$, although, for high-precision work, convergence is annoyingly slow unless one has at least $\sigma \gtrsim 2$. A globally convergent series was given by Helmut Hasse in 1930[10]:

$$
\zeta(s, q)=\frac{1}{s-1} \sum_{n=0}^{\infty} \frac{1}{n+1} \sum_{k=0}^{n}(-1)^{k}\left(\begin{array}{c}
n \\
k
\end{array}\right)(q+k)^{1-s}
$$

Date: 12 October 2006, revised 10 February 2007.

1991 Mathematics Subject Classification. 65B10 (primary), 11M35, 11Y35, 33F05, 68W25.

Key words and phrases. Polylogarithm, Hurwitz zeta function, algorithm, monodromy, series acceleration. 
Although this series is technically convergent everywhere, in practice, the convergence is abysmally slow on the critical strip. A not unreasonable algorithm may be given by considering a Taylor's series in $q$. Expanding about $q=0$, one obtains

$$
\zeta(s, q)=\frac{1}{q^{s}}+\sum_{n=0}^{\infty}(-q)^{n}\left(\begin{array}{c}
s+n-1 \\
n
\end{array}\right) \zeta(s+n)
$$

It is not hard to show that the sum on the right is convergent for $|q|<1$. Alternately, an expansion may be made about $q=1 / 2$ :

$$
\zeta\left(s, q+\frac{1}{2}\right)=\sum_{n=0}^{\infty}(-q)^{n}\left(\begin{array}{c}
s+n-1 \\
n
\end{array}\right)\left(2^{s+n}-1\right) \zeta(s+n)
$$

It may be shown that the above has a region of convergence $|q|<1 / 2$. Either of these expansions provide a computable expression for the Hurwitz zeta function that is convergent on the entire complex $s$-plane (minus, of course, $s=1$, and taking the appropriate limit, via l'Hopital's rule, when $s$ is a non-positive integer). The principal drawback to the use of these sums for high-precision calculations is the need for many high-precision evaluations of the Riemann zeta; the compute time can grow exponentially as the number of required digits is increased, especially when $q$ approaches the radius of convergence of the sums.

It is the poor performance of these sums that motivates the development of this paper. Since the Generalized Riemann Hypothesis can be phrased in terms of the values of the Hurwitz zeta function on the critical strip, it is of some interest to have a fast algorithm for computing high-precision values of this function in this region. There seems to be a paucity of work in this area. There is a discussion of fast algorithms for Dirichlet L-functions in [15] (the author has been unable to obtain a copy of this manuscript).

The Hurwitz zeta may be expressed in terms of the polylogarithm[12] (sometimes called the "fractional polylogarithm" to emphasize that $s$ is not an integer):

$$
\operatorname{Li}_{s}(z)=\sum_{n=1}^{\infty} \frac{z^{n}}{n^{s}}
$$

by means of Jonquière's identity[12, Section 7.12.2][11]

$$
\mathrm{Li}_{s}\left(e^{2 \pi i q}\right)+(-1)^{s} \mathrm{Li}_{s}\left(e^{-2 \pi i q}\right)=\frac{(2 \pi i)^{s}}{\Gamma(s)} \zeta(1-s, q)
$$

and so one might search for good algorithms for polylogarithms. Wood[19] provides a extensive review of the means of computing the polylogarithm, but limits himself to real values of $s$. Crandall[7] discusses evaluating the polylogarithm on the entire complex $z$ plane, but limits himself to integer values of $s$. Thus, there appears to be a similar paucity of general algorithms for the polylogarithm as well. The series defining the polylogarithm may be directly evaluated when $|z|<1$, although direct evaluation becomes quite slow when $|z| \gtrsim 0.95$ and $\sigma \lesssim 2$. There do not seem to be any published algorithms that may be applied on the critical strip.

The primary effort of this paper is to take the algorithm given by Borwein [4, 5], which is a simple Padé-approximant type algorithm, and generalize it to the polylogarithm. The result is a relatively small finite sum that approximates the polylogarithm, and whose error, or difference from the exact value, can be precisely characterized and bounded. Increased precision is easily obtainable by evaluating a slightly larger sum; one may obtain roughly $2 N$ to $4 N$ bits of precision by evaluating a sum of $N$ terms. The sum may be readily evaluated for just about any value $s$ on the complex $s$-plane. However, it is convergent only 
in a limited region of the $z$-plane, and specifically, is only convergent when

$$
\left|\frac{z^{2}}{z-1}\right|<4
$$

This is sufficient for evaluating the Hurwitz zeta for general complex $s$ and real $0<q<$ 1. Unfortunately, there does not appear to be any simple and efficient way of extending this result to the general complex $z$-plane, at least not for general values of $s$. By using duplication and reflection formulas, one may extend the algorithm to the entire complex $z$-plane; however, this requires the independent evaluation of equation 1.3

The development of this paper proceeds as follows. First, a certain specific integral representation is given for the polylogarithm. This representation is such that a certain trick, here called "the Borwein trick", may be applied, to express the integral as a polynomial plus a small error term. This is followed by a sketch of a generalization of the trick to the convergence acceleration of general oscillatory series. The next section selects a specific polynomial, and the error term of the resulting approximation is precisely bounded. This is followed by a brief review of the duplication formula for the Hurwitz zeta, and a short discussion of ways in which this numerical algorithm may be tested for correctness. This is followed by a detailed derivation of the monodromy group, and a discussion of Apostol's "periodic zeta function".

The algorithm has been implemented by the author using the Gnu Multiple Precision arithmetic library [8], and is available on request, under the terms of the LGPL license. The paper concludes with some intriguing images of the polylogarithm and the Hurwitz zeta function on the critical strip.

\section{The Polylogarithm}

The polylogarithm has a non-trivial analytic structure. For fixed $s$, the principal sheet has a branch cut on the positive real axis, for $1<z<+\infty$. The location of the branchpoint at $z=1$, as is always the case with branch points, is the cause of limited convergence of analytic series in the complex $z$-plane. This is noted here, as this branch point has a direct impact on the algorithm, preventing convergence in its vicinity. Besides this, the polylogarithm has many other interesting properties, which are not reviewed here.

The development of the algorithm requires the following integral representation.

Lemma 2.1. The polylogarithm has the integral representation

$$
L i_{s}(z)=\frac{z}{\Gamma(s)} \int_{0}^{1} \frac{|\log y|^{s-1}}{1-y z} d y
$$

Proof. This identity is easily obtained by inserting the integral representation of the Gamma function:

$$
\begin{aligned}
\operatorname{Li}_{s}(z) & =\frac{1}{\Gamma(s)} \sum_{n=1}^{\infty} \frac{z^{n}}{n^{s}} \int_{0}^{\infty} e^{-u} u^{s-1} d u \\
& =\frac{1}{\Gamma(s)} \sum_{n=1}^{\infty} z^{n} \int_{0}^{\infty} e^{-n t} t^{s-1} d t \\
& =\frac{1}{\Gamma(s)} \int_{0}^{\infty} t^{s-1} \sum_{n=1}^{\infty}\left(z e^{-t}\right)^{n} d t \\
& =\frac{1}{\Gamma(s)} \int_{0}^{\infty} t^{s-1}\left[\frac{z e^{-t}}{1-z e^{-t}}\right] d t
\end{aligned}
$$


and then finally substituting $t=-\log y$ in the last integral. Although this derivation casually interchanges the order of integration and summation, one may appeal to general arguments about analytic continuation to argue that the final result is generally valid, provided one is careful to navigate about the branch point at $z=1$.

A more sophisticated presentation of this theorem is given by Costin[6, Thm. 1].

\section{THE BORWEIN TRICK}

The Borwein trick uses the integral form to find a simple, finite sum that approximates the desired value arbitrarily well. The trick consists of two steps. The first is to write the above integral in the form

$$
\begin{aligned}
\xi(s, z) & =\frac{1}{f(1 / z)} \frac{z}{\Gamma(s)} \int_{0}^{1} \frac{f(y)|\log y|^{s-1}}{1-y z} d y \\
& =\operatorname{Li}_{s}(z)+\frac{1}{f(1 / z)} \frac{z}{\Gamma(s)} \int_{0}^{1} \frac{f(y)-f(1 / z)}{1-y z}|\log y|^{s-1} d y
\end{aligned}
$$

The second step is to find a sequence of polynomials $p_{n}(z)$ to be used for $f(z)$, such that the integral on the right hand side can be evaluated as a simple, finite sum, while the left hand side can be shown to bounded arbitrarily close to zero as $n \rightarrow \infty$. The right hand side may be easily evaluated, by employing the following theorem.

Lemma 3.1. Given a polynomial $p_{n}(y)$ of degree $n$, it can be shown that

$$
r_{n}(y)=\frac{p_{n}(y)-p_{n}(1 / z)}{1-y z}
$$

is again a polynomial in $y$, of degree $n-1$, for any constant $z$, provided that $z \neq 0$.

Proof. This may be easily proved, term by term, by noting that $\left(x^{n}-a^{n}\right) /(x-a)$, with $a=1 / z$, has the desired properties.

An explicit expression for $r_{n}(y)$ is needed. Write the polynomial as

$$
p_{n}(y)=\sum_{k=0}^{n} a_{k} y^{k}
$$

while for $r_{n}$ assume only a general series:

$$
r_{n}(y)=\sum_{k=0}^{\infty} c_{k} y^{k}
$$

Setting $y=0$, one immediately obtains $c_{0}=a_{0}-p_{n}(1 / z)$. Higher coefficients are obtained by equating derivatives:

$$
r_{n}^{(k)}(y)=\frac{1}{1-y z}\left[p_{n}^{(k)}(y)+k z r_{n}^{(k-1)}(y)\right]
$$

where $r_{n}^{(k)}(y)$ is the $k$ 'th derivative of $r_{n}$ with respect to $y$. Setting $y=0$ in the above, one obtains the recurrence relation $c_{k}=a_{k}+z c_{k-1}$ which is trivially solvable as

$$
c_{k}=z^{k}\left[-p_{n}\left(\frac{1}{z}\right)+\sum_{j=0}^{k} \frac{a_{j}}{z^{j}}\right]
$$

From this, it is easily seen that $c_{n}=0$ and more precisely $c_{n+m}=0$ for all $m \geq 0$. This confirms the claim that $r_{n}(y)$ is a polynomial of degree $n-1$ in $y$.

This is immediately employed in equation 3.1 to obtain 


$$
\xi(s, z)=\mathrm{Li}_{s}(z)+\frac{z}{p_{n}(1 / z)} \sum_{k=0}^{n-1} \frac{c_{k}}{(k+1)^{s}}
$$

To obtain a good approximation for $\operatorname{Li}_{s}(z)$, one needs to find a polynomial sequence such that $\xi$ goes to zero as $n \rightarrow \infty$ for the domain $(s, z)$ of interest. That is, one seeks to make

$$
\xi(s, z)=\frac{1}{p_{n}(1 / z)} \frac{z}{\Gamma(s)} \int_{0}^{1} \frac{p_{n}(y)|\log y|^{s-1}}{1-y z} d y
$$

as small as possible. One possible sequence, based on the Bernoulli process or Gaussian distribution, is explored in a subsequent section.

\section{Convergence Acceleration of Oscillatory Series}

For the case of $z=-1$ and $p_{n}(y)=y^{n}$, the technique developed above corresponds to Euler's method for the convergence acceleration of an alternating series[1, eqn 3.6.27]; a particularly efficient algorithm for Euler's method is given by van Wijngaarden[18]. Polynomials that provide much more powerful series acceleration are discussed by Cohen etal[17], but again in the context of $z=-1$; these include the Tchebysheff orthogonal polynomials considered by Borwein, which do a rather good job of minimizing the integrand of equation 3.8 when $z=-1$. In a certain sense, an alternating series can be considered to be a series from which an explicit factor of $z^{n}$ has been factored out, for the very special case of $z=-1$. Thus, one is lead to ask if one can find series acceleration methods for more general oscillatory series, where the oscillatory component can be thought of as going as $z^{n}$ for some root of unity $z$.

The Euler method applied to the Hurwitz zeta function essentially leads to the Hasse series 1.2 This may be seen by noting that the inner sum is just a forward difference:

$$
\sum_{k=0}^{n}(-1)^{k}\left(\begin{array}{c}
n \\
k
\end{array}\right)(q+k)^{1-s}=\triangle^{n} q^{1-s}
$$

where $\triangle$ is the forward difference operator. The Hasse series has improved convergence in that the result is globally convergent, whereas the traditional series representation of equation 1.1 is not. However, a quick numerical experiment will show that the Hasse series does not converge rapidly, especially in the critical strip.

The poor convergence of these traditional techniques motivates the development of this paper. Strictly speaking, the slow convergence can be attributed to the fact that the naive series representations 1.1 or 1.4 are not strictly alternating series, as would be required to justify the application of the Euler method. Instead, the series are a superposition of oscillatory terms; for the polylogarithm, an explicit oscillation coming from $z^{n}=|z|^{n} e^{\text {in arg } z}$ and a slower oscillation coming from $n^{-s}=n^{-\sigma} e^{-i \tau \log n}$. Thus, what one needs is a theory of sequence acceleration not for alternating series, but for oscillatory series. The polylogarithm approximation represents the ad-hoc development of a special case for such a general theory.

A loose sketch of a possible general theory is presented below. Suppose that $c_{n}$ is some more or less arbitrary sequence of numbers, oscillatory in $n$, and that one wishes to compute the sum

$$
S=\sum_{\substack{n=0 \\ 5}}^{\infty} c_{n}
$$


but that one wants to apply convergence acceleration techniques to its evaluation. If the leading component of oscillation is $c_{n} \sim \cos n \theta$, then one makes the ansatz that there must exist some dual series $s_{n}$ oscillating as $s_{n} \sim \sin n \theta$ to give $e_{n}=c_{n}+i s_{n} \sim e^{i n \theta}$. One may then consider a sum of $a_{n}=e^{i n(\pi-\theta)} e_{n}$ and consider the summation of an alternating series

$$
\sum_{n=0}^{\infty}(-1)^{n} a_{n}
$$

By construction, the $a_{n}$ are now presumed to be relatively tame, so that series acceleration techniques can be applied. This trick may be applied to any oscillatory sequence; one need not know a precise or a priori value for $\theta$; one need only guess at a reasonable value, based on the data at hand.

Alternately, one may consider replacing the forward difference operator $\triangle b_{n}=b_{n+1}-$ $b_{n}$ by $\triangle_{q} b_{n}=b_{n+1}-q b_{n}$. Straightforward manipulations lead to

$$
\sum_{m=0}^{\infty}\left(\frac{z}{1-q z}\right)^{m+1} \triangle_{q}^{m} b_{0}=\sum_{n=0}^{\infty} z^{n} b_{n}
$$

with the traditional Euler's series regained by taking $q=1$ and $z=-1$. Assuming that the oscillatory part $z$ is known, or can be approximately guessed at from the period of the oscillation, then choosing $q=-1 / z$ should make the left hand side an accelerated series for the right hand side. However, there are more powerful techniques.

Suppose one is interested in the sum

$$
S(z)=\sum_{n=0}^{\infty} z^{n} b_{n}
$$

In general, one is interested in general sequences $b_{n}$. Suppose, however, that the $b_{n}$ are expressible as moments, so that

$$
b_{n}=\int_{0}^{1} y^{n} g(y) d y
$$

for some function $g(y)$. Since $g(y)$ is still fairly general (subject to constraints discussed below), this assumption does not overly restrict the $b_{n}$. If the $b_{n}$ are expressible as moments, then one has

$$
S(z)=\int_{0}^{1} \frac{g(y)}{1-y z} d y
$$

Applying the Borwein trick, one obtains

$$
S(z)=S_{n}(z)+\xi(z)
$$

with

and

$$
S_{n}(z)=\frac{1}{p_{n}\left(\frac{1}{z}\right)} \int_{0}^{1} \frac{p_{n}(y)-p_{n}\left(\frac{1}{z}\right)}{1-y z} g(y) d y
$$

$$
\xi_{n}(z)=\frac{1}{p_{n}\left(\frac{1}{z}\right)} \int_{0}^{1} \frac{p_{n}(y) g(y)}{1-y z} d y
$$

The goal is to show that the $S_{n}(z)$ are easily evaluated, while also showing that the $\xi_{n}(z)$ are arbitrarily small. The first is easy enough: using equations 3.2 and 3.4 one has

$$
S_{n}(z)=\frac{1}{p_{n}\left(\frac{1}{z}\right)} \sum_{k=0}^{n-1} c_{k} b_{k}
$$


With a suitable choice of $p_{n}(z)$, the coefficients $c_{k}$ are presumably easy to evaluate. To show that the $S_{n}(z)$ is really a series acceleration for the geometric series 4.1, one must show that $\left|\xi_{n}(z)\right|$ is bounded. If $g(y) \geq 0$, then

$$
\left|\xi_{n}(z)\right| \leq\left|\frac{p_{n}\left(y_{0}\right)}{p_{n}\left(\frac{1}{z}\right)}\right||S(z)|
$$

where $y_{0}$ is the point on the unit interval where $p_{n}(y)$ assumes its maximum. Provided that one can find a polynomial sequence such that

$$
\left|\frac{p_{n}\left(y_{0}\right)}{p_{n}\left(\frac{1}{z}\right)}\right| \sim A^{-n}
$$

for some number $A>|1 / z|$, then one has that the series $S_{n}(z)$ converges to $S(z)$ more rapidly than the simple partial sums $\sum_{k=0}^{n} z^{k} b_{k}$ of equation 4.1 .

Note that the above is a proof of a series acceleration method for a more-or-less general sequence of $b_{n}$, and is in no way particular to the polylogarithm or the Hurwitz zeta. The only ingredients of the proof were that the $b_{n}$ are "well-behaved" - in this case, being expressible as moments of a rather general function $g(y)$. For the proof to hold, $g(y)$ needs to be (mostly) positive, and integrable; it need not be analytic, differentiable or even continuous. It needs to be "mostly" positive only so that the bound on the integrand, $p_{n}(y) g(y) \leq\left|p_{n}\left(y_{0}\right)\right| g(y)$ can be established. Thus, one might hope that the acceleration method might work, even for those general cases where $g(y)$ is not explicitly known, but the $b_{n}$ are somehow "reasonable".

For the special case of $z=-1$, i.e. for the case of an alternating series, Cohen et al.[17] suggest some remarkably strongly converging polynomials, with values of $A$ from 5.8 to as much as 17.9. The generalization of those results to arbitrary $z$ is not immediately apparent, but is surely possible.

There is another, possibly fruitful direction one might take. This hinges on noting that the integrands above take the general form of a Padé approximation, and one proceeds from there.

\section{The GaUsSian Distribution}

Returning to the polylogarithm, the task at hand is to find a polynomial sequence that is suitable for establishing a tight bound on the error term $\xi$. A good bound minimizes the integrand, while also maximizing the value of $\left|p_{n}(1 / z)\right|$. In order to suppress the logarithmic branch point at $y=0$ in the integrand of 3.8 , this section will consider the polynomial sequence $p_{n}(y)=y^{a}(1-y)^{n-a}$, a sequence which goes over to the Gaussian distribution for large $n$.

Consider first the polynomial sequence $p_{2 n}(y)=y^{n}(1-y)^{n}$. It becomes sharply peaked at $y=1 / 2$, and subtends a diminishingly small area. For large values of $n$, this polynomial sequence approximates a Gaussian:

$$
p_{2 n}(y)=\frac{1}{4^{n}} e^{-4 n(y-1 / 2)^{2}}\left[1+2\left(y-\frac{1}{2}\right)^{2}+O\left(\left(y-\frac{1}{2}\right)^{4}\right)\right]
$$

Using this in the integral 3.8, and assuming that the real part of $z$ is not positive, it is not hard to deduce the very crude estimate

$$
\xi(s, z) \sim\left(\frac{z^{2}}{4(z-1)}\right)^{n} \frac{z}{\Gamma(s)}
$$


which confirms that $\xi(s, z)$ does indeed get suitably small for a certain region in the complex $z$-plane. However, in order for equation 3.7 to be useful computationally, an upper bound on the value of $\xi$ needs to be given, as a function of $z$ and $n$. This bound is derived in the next section.

The polynomial coefficients are easily obtained, and are

$$
\begin{aligned}
a_{0} & =a_{1}=\cdots=a_{n-1}=0 \\
a_{n+k} & =(-1)^{k}\left(\begin{array}{c}
n \\
k
\end{array}\right) \quad \text { for } 0 \leq k \leq n
\end{aligned}
$$

This leads to

$$
\operatorname{Li}_{s}(z)=-\frac{z^{2 n+1}}{(z-1)^{n}} \sum_{k=0}^{2 n-1} \frac{c_{k}}{(k+1)^{s}}+\xi(s, z)
$$

where the $c_{k}$ are given by

$$
c_{k}=z^{k}\left[-\left(\frac{z-1}{z^{2}}\right)^{n}+\frac{1}{z^{n}} \sum_{j=0}^{k-n}(-1)^{j}\left(\begin{array}{c}
n \\
j
\end{array}\right) \frac{1}{z^{j}}\right]
$$

where the summation above is to be understood to be zero when $k<n$. The above summations can be re-organized into the more suggestive form

$$
\mathrm{Li}_{s}(z)=\sum_{k=1}^{n} \frac{z^{k}}{k^{s}}+\frac{1}{(1-z)^{n}} \sum_{k=n+1}^{2 n} \frac{z^{k}}{k^{s}} \sum_{j=0}^{2 n-k}(-z)^{j}\left(\begin{array}{c}
n \\
j
\end{array}\right)+\xi(s, z)
$$

The error term $\xi(s, z)$ is negligible for only a very specific area of the complex $z$-plane. The region where $\xi$ may be ignored as a small error is derived in the next section. Substituting $z=-1$ in the above formulas agrees with expressions previously given by Borwein [4].

The above formula has been implemented using the Gnu Multiple Precision arithmetic library (GMP) [8], and has been numerically validated for correctness in several different ways. The source code, under the license terms of the LGPL, may be obtained by contacting the author.

\section{BOUND ON THE ERROR TERM}

In order for equation 5.5 to be useful computationally, an upper bound on the value of $\xi$ as a function of $n$ needs to be given. To compute the polylogarithm to some desired precision, one infers a suitable value of $n$ based on this bound. However, to achieve this desired precision, one must not only choose $n$ large enough, but one must also maintain a somewhat larger number of digits in the intermediate terms, as the appearance of the binomial coefficient in the equation 5.5 implies that intermediate terms can become quite large, even while mostly cancelling. This section derives an upper bound on the size of $\xi$, and briefly discusses the issue of the required precision in intermediate terms.

The general behavior of the integrand appearing in equation 3.8 depends on the whether $\Re s \geq 1$ or not; estimates are presented for these two cases. Writing $s=\sigma+i \tau$ for real $\sigma$ and $\tau$, and assuming $\sigma \geq 1$ and choosing $n$ so that $\sigma \leq n$, one has

$$
\begin{aligned}
\left.\left|p_{2 n}(y)\right| \log y\right|^{s-1} \mid & \leq p_{2 n}(y)|\log y|^{\sigma-1} \\
& =|y(1-y) \log y|^{\sigma-1}(y(1-y))^{n-\sigma+1}
\end{aligned}
$$

Each part of the right hand side is bounded by a Gaussian, and since the product of Gaussians is a Gaussian, so is the entire expression. From the Stirling approximation, one has 
the well-known identity

$$
(y(1-y))^{a} \leq \frac{1}{4^{a}} \exp -4 a\left(y-\frac{1}{2}\right)^{2}
$$

The other part is also bounded by a Gaussian

$$
|y(1-y) \log y|^{a} \leq\left|y_{0}\left(1-y_{0}\right) \log y_{0}\right|^{a} \exp \left(-\frac{a\left(y-y_{0}\right)^{2}}{2 y_{0}\left(1-2 y_{0}\right)}\right)
$$

which is centered on the maximum of $\left|y_{0}\left(1-y_{0}\right) \log y_{0}\right|$, that is, at $y_{0}=0.23561058253 \ldots$, where $y_{0}$ is the solution to

$$
0=\frac{d}{d y} y(1-y)|\log y|
$$

Curiously, the numerical value of the factor in the Gaussian is $1 / 2 y_{0}\left(1-2 y_{0}\right)=4.013295587 \ldots$

To evaluate the integral in 3.8, one also needs a bound on the denominator. This is furnished by

$$
\left|\frac{1}{1-y z}\right| \leq C(z)= \begin{cases}1 & \text { if } \Re z \leq 0 \\ \frac{1}{1-z} \mid & \text { if } 0<\Re z \text { and }|z|<1 \\ \left|\frac{z}{\mathfrak{I} z}\right| & \text { if } 0<\Re z \text { and }|z|>1\end{cases}
$$

Thus, the integrand is bounded by a Gaussian. Multiplying the two Gaussians, completing the square, and evaluating the resulting integral is a bit tedious. The result is that

$$
\left|\int_{0}^{1} \frac{p_{2 n}(y)|\log y|^{s-1}}{1-y z} d y\right| \leq C(z) \frac{\left|4 y_{0}\left(1-y_{0}\right) \log y_{0}\right|^{\sigma-1}}{4^{n}} G(s)
$$

The constant may be taken as $\left|4 y_{0}\left(1-y_{0}\right) \log y_{0}\right|=1.041381965 \ldots$. The factor $G(s)$ is bounded by

$$
\begin{aligned}
G(s) & \leq \sqrt{\frac{\pi}{4 n}} \exp -\left(1-2 y_{0}\right)^{2}(\sigma-1) \frac{8 y_{0}\left(1-2 y_{0}\right)}{8 y_{0}\left(1-2 y_{0}\right)+\frac{\sigma-1}{n-\sigma+1}} \\
& \leq \sqrt{\frac{\pi}{4 n}} \exp -\left(1-2 y_{0}\right)^{2} \frac{\sigma-1}{\sigma} \\
& \leq 1
\end{aligned}
$$

when $1 \leq \sigma$. Useful for estimation is that $8 y_{0}\left(1-2 y_{0}\right)=0.996687115 \ldots$.. Combining these results, one obtains

$$
|\xi(s, z)| \leq \frac{1}{4^{n}}\left|\frac{z^{2}}{z-1}\right|^{n}\left|\frac{z}{\Gamma(s)}\right| C(z) G(s)(1.0414 \ldots)^{\sigma-1}
$$

for the case where $1 \leq \sigma$ and $n$ chosen so that $\sigma \leq n$.

The case where $\sigma \leq 1$ may be evaluated as follows. One writes

$$
\begin{aligned}
\left.\left|p_{2 n}(y)\right| \log y\right|^{s-1} \mid & \leq p_{2 n}(y)|\log y|^{\sigma-1} \\
& =\left|\frac{1-y}{\log y}\right|^{1-\sigma} y^{n}(1-y)^{n+\sigma-1} \\
& \leq y^{n}(1-y)^{n+\sigma-1}
\end{aligned}
$$


The integrand may now be recognized as the Beta function, so that

$$
\left|\int_{0}^{1} \frac{p_{2 n}(y)|\log y|^{s-1}}{1-y z} d y\right| \leq C(z) \frac{\Gamma(n+1) \Gamma(n+\sigma)}{\Gamma(2 n+\sigma+1)}
$$

In the region where $|\sigma| \ll n$, one may approximate

$$
\frac{\Gamma(n+1) \Gamma(n+\sigma)}{\Gamma(2 n+\sigma+1)} \leq \frac{2^{1-\sigma}}{4^{n}}
$$

Combining the various pieces, one obtains a result remarkably similar to the previous one; namely, when $\sigma \leq 1$ but $n$ is such that $|\sigma| \ll n$, one has

$$
|\xi(s, z)| \leq \frac{1}{4^{n}}\left|\frac{z^{2}}{z-1}\right|^{n}\left|\frac{z}{\Gamma(s)}\right| 2^{1-\sigma} C(z)
$$

For evaluations on the critical line $\sigma=1 / 2$, one needs a good estimate for $|\Gamma(s)|^{-1}$, which can become quite large as the imaginary part of $s$ increases. A useful estimate for $|\Gamma(s)|^{-1}$ is given by Borwein [5, 4], for the case where $\sigma \geq-m+1 / 2$ :

$$
\begin{aligned}
\frac{1}{|\Gamma(s)|} & =\frac{1}{|\Gamma(\sigma)|} \sqrt{\prod_{k=0}^{\infty}\left(1+\frac{\tau^{2}}{(\sigma+k)^{2}}\right)} \\
& \leq \frac{1}{|\Gamma(\sigma)|} \sqrt{\frac{\sinh \tau \pi}{\tau \pi} \prod_{k=0}^{m}\left(1+\frac{\tau^{2}}{\left(\frac{1}{2}+k\right)^{2}}\right)}
\end{aligned}
$$

A remarkable side-effect of the estimation is that, for $s$ equal to negative integers, one has that $\Gamma(s)$ is infinite, thus implying that the error term is zero. This somewhat surprising result corresponds to the fact that $\mathrm{Li}_{-n}(z)$ has an exact expression as a ratio of two polynomials, the denominator being of degree $n+1$. Indeed, setting $s=0$ and so $n=1$ into equation 5.5 gives the exact result

$$
\operatorname{Li}_{0}(z)=\frac{z}{1-z}
$$

while for $s=-1$, one must use $n=2$, to obtain the exact result

$$
\mathrm{Li}_{-1}(z)=\frac{z}{(1-z)^{2}}
$$

The generic form for the polylogarithm at the negative integers is

$$
\mathrm{Li}_{-n}(z)=(-1)^{n} \sum_{k=0}^{n}(-1)^{k} \frac{k !}{(1-z)^{k+1}}\left\{\begin{array}{c}
n+1 \\
k+1
\end{array}\right\}
$$

where $\left\{\begin{array}{l}n \\ k\end{array}\right\}$ denotes the Stirling numbers of the second kind.

In general, the formula 5.5 seems to have the curious property of always evaluating to exactly the same rational function whenever $s$ is a non-positive integer, and $-s<n$. That is, for fixed non-positive integer $s$, the sum is independent of $n$, provided that $n$ is large enough. Thus, this polynomial approximation has the pleasing result of giving exact answers in the case when an exact polynomial answer is possible.

For the general case, one may conclude that the estimate 5.5 can be effectively applied whenever

$$
\left|\frac{z^{2}}{z-1}\right|<4
$$


To obtain a value of the polylogarithm to within some given numerical precision, one must invert the formulas 6.3 or 6.4 solving for the value of $n$ which is to be used in equation 5.5. To obtain a fixed number of digits of precision, one must carry out intermediate calculations with at least that many digits of precision. In fact, one must have more. The appearance of the binomial coefficient in equation 5.5 is the problem. Since $2^{n}>\left(\begin{array}{c}n \\ j\end{array}\right)$, one concludes that $n$ additional binary bits of precision are needed with which to carry out the calculations. As a result, it becomes difficult to implement the algorithm robustly using only double-precision arithmetic. For values of $z$ near $z=-1$, the algorithm is well-enough behaved; however, round-off errors significantly disturb the calculations as $z$ approaches +1 . This trouble with precision can be at least partly alleviated by making use of the duplication formula, as discussed in the next section.

\section{Performance}

The performance of the Borwein algorithm appears to be very good. In fact, it appears to have better performance than the direct summation formula 1.4 in all cases, even when direct summation might seem to have an advantage. The figures 7.1 and 7.2 compare the performance of actual implementations.

The first figure shows "cold cache" performance, measured in seconds, as compared to the number of desired decimal places of precision. The figure is termed "cold cache", in recognition of the fact that some constants may be pre-computed. For example, if $s$ is held fixed, while $z$ is varied, then the values of $n^{-s}$ appearing in both the direct sum and the Borwein algorithm may be computed once, and then re-used for subsequent calculations. As the figures indicate, computing $n^{-s}$ can be very expensive for general complex-valued $s$, and so the caching strategy offers a big gain when $s$ is held constant.

As the "cold cache" figure demonstrates, the Borwein algorithm is faster than direct summation. The problem with direct summation is that it requires more values of $n^{-s}$ to be computed to achieve comparable precision.

As the "warm cache" figure shows, the Borwein algorithm will still win against direct summation, even when the values of the $n^{-s}$ are pre-computed. Even when these are pre-computed, and can be pulled from the cache, direct summation still requires more operations.

A different but equally dramatic set of results obtain, when one compares the performance of the Borwein algorithm applied to the Hurwitz zeta function, as compared to the use of the Taylor's expansion 1.3 for the Hurwitz zeta. In this case, as the figures show, the Taylor's series outperforms the Borwein algorithm by a constant factor of three, when both algorithms use pre-computed constants. However, the cost of pre-computing these constants skyrockets for the Taylor's series, making it unattractive as the number of required decimal places increases.

\section{DUPLiCATION FORMUla}

The region of applicability of the algorithm may be extended by making use of the duplication formula for the polylogarithm or periodic zeta. The duplication formula, or more generally, the multiplication theorem, is an extension of the well-known Legendre duplication formula for the Gamma function[1] (6.1.18),(6.3.8)(23.1.10)], into the domain of the polylogarithm. 
FIGURE 7.1. Polylogarithm cold cache performance

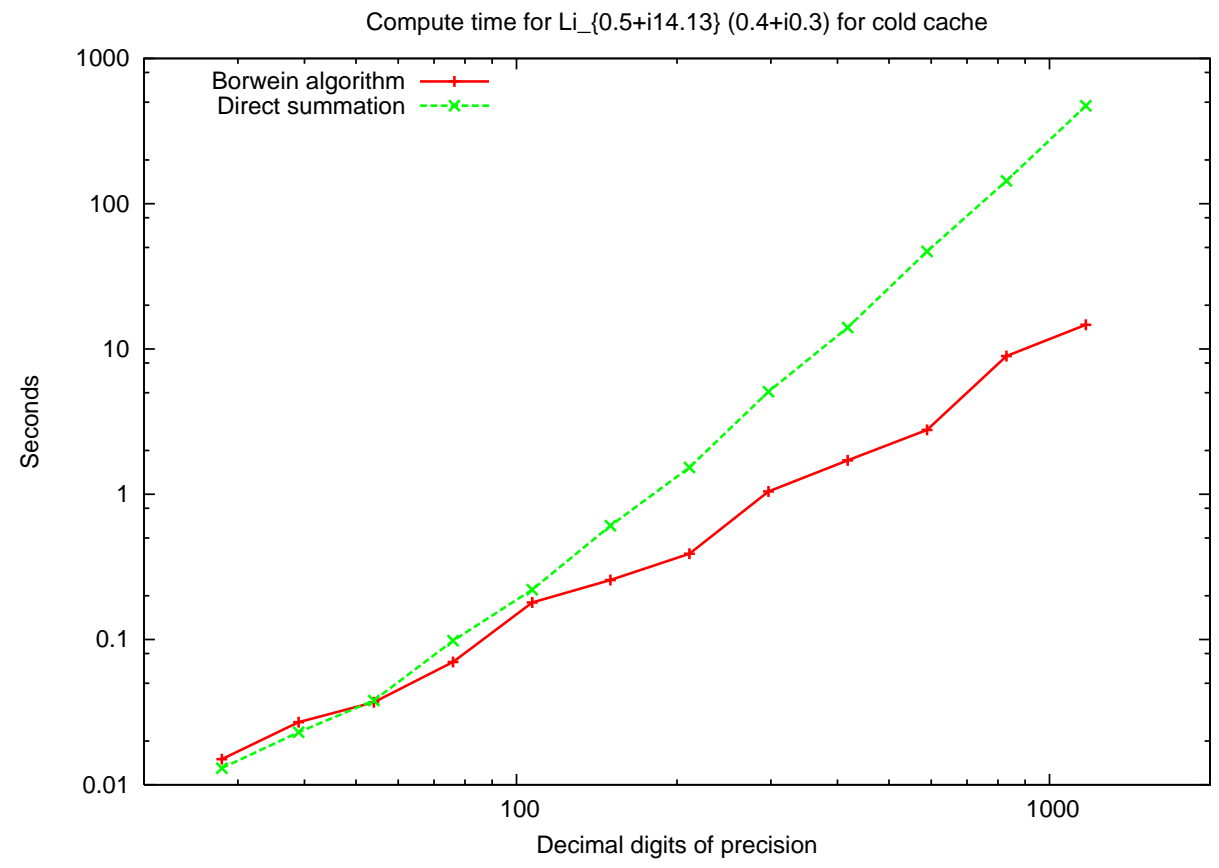

This figure shows the time, in seconds, needed to compute the polylogarithm to the indicated number of decimal places of precision. The values chosen for evaluation are $s=0.5+i 14.13$, which is very near to one of the Riemann zeroes, and $z=0.4+i 0.3$, which is relatively near to the origin: thus, one might have hoped that, for such a small

value $(|z|=0.5)$, direct summation might compete favorably against the Borwein algorithm, as the point is closer to +1 than to -1 . Nevertheless, $\left|z^{2} /(z-1)\right| \approx 0.373$ for this point, and the Borwein algorithm wins.

The primary contribution to the calculation of either value is the evaluation of $n^{-s}$ in the summations. If these are pre-computed, the Borwein algorithm still wins, as shown in the next figure. All calculations were performed on a contemporary desktop computer, using the GMP library; the algorithms were implemented in the $\mathrm{C}$ programming language. The power-laws that seem to apply to the slopes are $T \approx O\left(D^{1.8}\right)$ and $T \approx O\left(D^{2.8}\right)$, respectively, for the elapsed time $T$ to compute $D$ digits. Note that the actual performance depends on the underlying implementation of log, exp, sin, atan, sqrt, gamma and the like; the actual implementation used here is fairly naive and untuned.

Thus, for example, the formula5.5 together with the error bounds 6.3 6.4 allow $F(q ; s)=$ $\mathrm{Li}_{s}\left(e^{2 \pi i q}\right)$ to be computed for real $q$ in region $1 / 4 \leq q \leq 3 / 4$. To obtain values on the region $0<q<1 / 4$, one applies the duplication formula

$$
F(q ; s)=2^{1-s} F(2 q ; s)-F\left(q+\frac{1}{2} ; s\right)
$$

recursively until one obtains values of $q \geq 1 / 4$. Rearranging terms, one obtains a similar formula for iterating values of $q>3 / 4$ until they are less than or equal to $3 / 4$. Thus, 
FIGURE 7.2. Polylogarithm warm cache

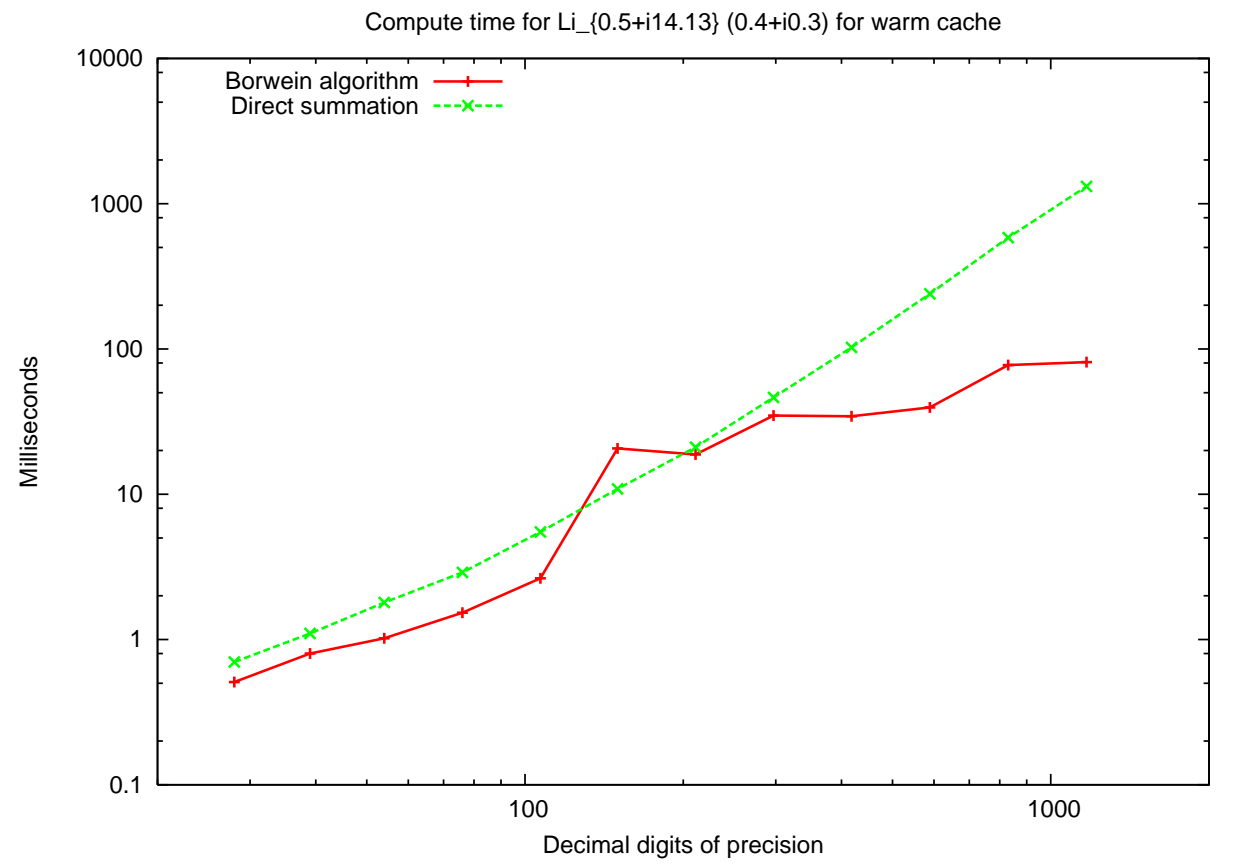

This graph compares the compute times for the Borwein algorithm and the direct summation of the polylogarithm, for a "warm cache". That is, an array of values of $n^{-s}$ were pre-computed, prior to beginning the timing measurements. These pre-computed values were used in the Borwein and direct sums. Despite the use of such cached, pre-computed values, the Borwein algorithm still wins. This graph uses the same $s$ and $z$ values as in the previous graph.

The power-law performance appears to be about $T \approx O\left(D^{1.4}\right)$ and $T \approx O\left(D^{2.1}\right)$ respectively, to compute $D$ decimal digits in time $T$.

as $q$ approaches 0 or 1, the algorithm requires more time, but only logarithmically so, as $-\log _{2} q$ or $-\log _{2}(1-q)$ as the case may be.

The duplication formula follows from a general $p$-adic identity

$$
\sum_{m=0}^{p-1} F\left(\frac{m+q}{p} ; s\right)=p^{1-s} F(q ; s)
$$

which is valid for any positive integer $p$. One of many ways of obtaining this formula is by noting that the function $F(s ; q)$ is an eigenvalue of the $p$-adic Bernoulli transfer operator associated with eigenvalue $p^{1-s}[16]$.

The equivalent formulas[12, Sections 7.3.1, 7.12.1] for the polylogarithm are

$$
\mathrm{Li}_{s}(z)+\mathrm{Li}_{s}(-z)=2^{1-s} \mathrm{Li}_{s}\left(z^{2}\right)
$$

whereas the $p$-adic version has the appearance of a Gauss sum:

$$
\sum_{m=0}^{p-1} \operatorname{Li}_{s}\left(z e^{2 \pi i m / p}\right)=p^{1-s} \operatorname{Li}_{s}\left(z^{p}\right)
$$


FIGURE 7.3. Hurwitz zeta cold cache performance

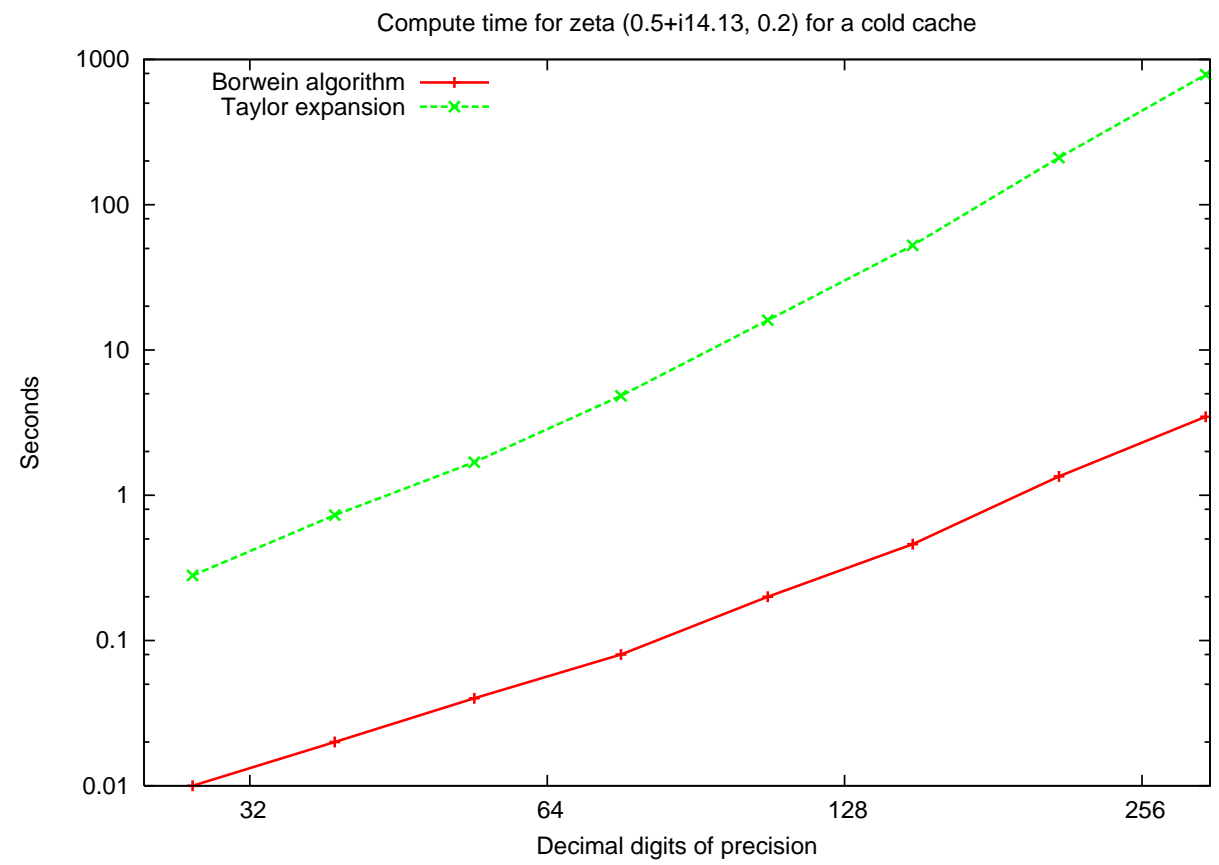

This figure compares the performance of the Borwein algorithm to the evaluation of the Taylor expansion for the Hurwitz zeta. The vales computed are for $s=0.5+i 14.13$ and $q=0.2$. For such a small value of $q$, one might have hoped that the Taylor expansion might converge quickly. This appears to not be the case, as the binomial coefficients can grow to be quite large, and thus force many terms to be summed.

The figure is for a "cold cache", assuming that no constants have been pre-computed. The lines are not parallel, with the Taylor expansion getting progressively worse. The Borwein algorithm can be seen to be more than an order of magnitude faster for lower precisions, and more than two orders of magnitude faster for the higher precisions.

The power-law performance appears to be about $T \approx O\left(D^{2.5}\right)$ and $T \approx O\left(D^{3.5}\right)$ respectively, to compute $D$ decimal digits in time $T$.

The application of the duplication formula, together with the inversion relation 1.5 can be used to extend the evaluation of the polylogarithm to the entire complex plane. For numerical work, both formulas must be applied, one alone is not enough. Consider first the application of the duplication formula only. It may be used to take points that are near to $z=1$, and map them further away from $z=1$, into the convergent region for the Borwein polynomial. Repeated application allows arbitrarily close approach to $z=1$ from the left-hand side. The resulting region of convergence is kidney-shaped, with the cusp of the kidney at $z=1$, and the kidney containing the unit disk $|z| \leq 1$. The precise shape of the kidney depends on the number of terms one wishes to use in the polynomial approximation. The shape that can be achieved while still maintaining good running time is shown in figure 4. However, as can be seen, this strategy barely penetrates the $\Re z>1$ region.

To extend the algorithm to the entire complex $z$-plane, one must make use of the Jonquière's inversion formula[14, pp 27-32] 
FIGURE 7.4. Hurwitz zeta warm cache performance

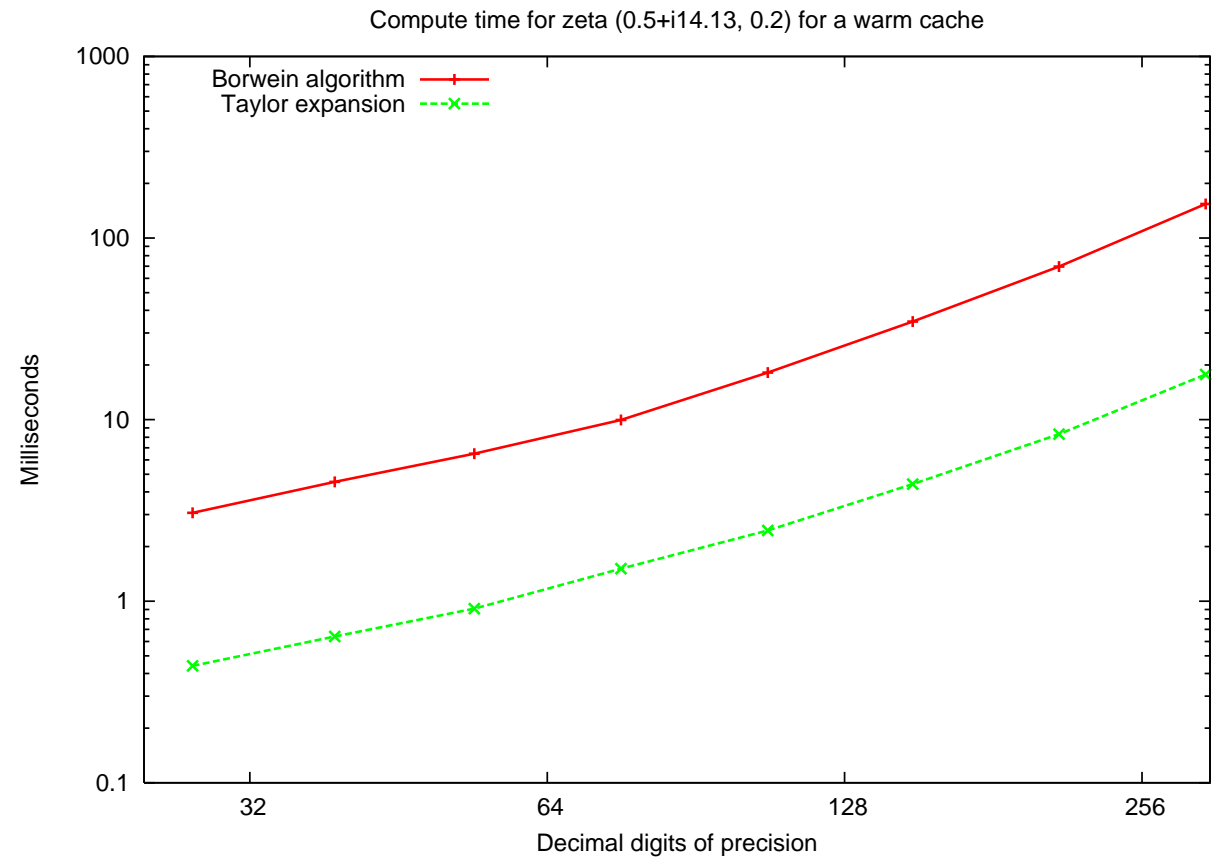

This figure compares the evaluation times for the Borwein algorithm, and the Hurwitz zeta Taylor's expansion, for the "warm cache" scenario. In this case, it is recognized that if $s$ is assumed to be held fixed, then the values of $n^{-s}$ appearing in the Borwein algorithm may be pre-computed. Similarly, the values of $\zeta(s+n)$ and the binomial coefficients $\left(\begin{array}{c}s+n-1 \\ n\end{array}\right)$ appearing in the Taylor's series may be pre-computed. In this case, the

Taylor's series evaluation appears to be about eight times faster.

The power-law performance appears to be about $T \approx O\left(D^{1.7}\right)$ and $T \approx O\left(D^{1.6}\right)$ respectively, to compute $D$ decimal digits in time $T$.

Performing the pre-computations can be terribly expensive. For example, for 300 decimal places of precision, the pre-computations take 13 minutes. The break-even point, where both algorithms are equally fast at fixed $s$, when including the pre-computation overhead, occurs at about 600 evaluations of 300 decimal places. Unless one is making a very large number of evaluations at fixed $s$, the pre-computation needed for the Taylor's expansion becomes prohibitively expensive as the number of desired decimal places goes up.

$$
e^{-i \pi s / 2} \operatorname{Li}_{s}(z)+e^{i \pi s / 2} \operatorname{Li}_{s}\left(\frac{1}{z}\right)=\frac{(2 \pi)^{s}}{\Gamma(s)} \zeta\left(1-s, \frac{\log z}{2 \pi i}\right)
$$

together with some sort of independent means of evaluating the Hurwitz zeta function. The Taylor expansion 1.3 is particularly well-suited, as it is rapidly convergent in the vicinity of $z=1$. Specifically, it is convergent when $|\log z|<2 \pi$, although the region of acceptable numerical convergence is smaller, roughly $|\log z|<\pi$. Either way, this encompasses a rather large region in the vicinity of $z=1$, which is exactly the region where the Borwein algorithm has trouble. The inversion formula is used to move points $|z|>1$ from outside 
of the unit circle, to the inside. Not all points inside the unit circle are directly accessible to the Borwein algorithm; the duplication formula must still be used to handle points in the disk interior that are near $z=1$. Similarly, points with very large $z$, such as those for which $|z|>e^{2 \pi}$, require the use of the duplication formula to be moved into the region of convergence for the Hurwitz Taylor series. A typical view of the polylogarithm on the complex $z$-plane is shown in figure. .5.

As a practical computational matter, rather than using 8.1 it seems to be more convenient to use the formula

$$
\operatorname{Li}_{1-s}(z)=\frac{\Gamma(s)}{(2 \pi)^{s}}\left[e^{i \pi s / 2} \zeta\left(s, \frac{\log z}{2 \pi i}\right)+e^{-i \pi s / 2 \zeta}\left(s, 1-\frac{\log z}{2 \pi i}\right)\right]
$$

Although this is equivalent to the equation 8.1 immediately above, it correctly captures the appropriate branch of the logarithm that should be used: equation 8.2 works well for values of $s$ in both the upper and lower half-planes, whereas equation 8.1 is more difficult to correctly evaluate in the lower half $s$-plane. In either case, one must use the logarithm with an unusual branch cut, taking it to run from $z=0$ to the right along the positive real axis, as opposed to the usual cut taken for the logarithm.

It is of some curiosity to note that the sheet that is forced takes the form

$$
\zeta(s, q)+e^{-i \pi s} \zeta(s, 1-q)=\sum_{n=-\infty}^{+\infty} \frac{1}{(n+q)^{s}}
$$

which is also noted by Costin[6, eqn 14] as a Mittag-Leffler type decomposition. A general discussion of the monodromy is given in a later section.

\section{Testing And VALidation}

The correctness of a given numerical implementation can be validated in a number of ways. For non-positive integer $s$, one has the exact rational functions previously mentioned. For positive integer $n$, one has the relationship

$$
\mathrm{Li}_{n}\left(e^{2 \pi i q}\right)+(-1)^{n} \mathrm{Li}_{n}\left(e^{-2 \pi i q}\right)=-\frac{(2 \pi i)^{n}}{n !} B_{n}(q)
$$

where the $B_{n}(x)$ are the Bernoulli polynomials. For $z=-1$, one regains the Riemann zeta function $\zeta(s)$ :

$$
\operatorname{Li}_{s}(-1)=\frac{1}{2^{1-s}-1} \zeta(s)
$$

For $|z|<1$, the defining series 1.4 is explicitly convergent, and may be directly summed, thus offering a fourth check of the correctness of an implementation. Finally, the multiplication theorem can be used to check for consistency. Each of these quantities can be computed by independent algorithms, and thus be used to validate the correctness of a polylogarithm implementation.

\section{BRANCH POINTS AND MONODROMY}

The principal sheet of the polylogarithm has a branch point at $z=1$, and by convention, a branch cut is placed along the positive real $z$-axis, extending to the right. As one moves off of the principal sheet, one discovers that there is another branch point at $z=0$. The resulting monodromy group is generated by two elements, acting on the covering space of the bouquet $S^{1} \vee S^{1}$ of homotopy classes of loops in $\mathbb{C} \backslash\{0,1\}$ passing around the branch points $z=0$ or $z=1$. The author is not aware of any simple published discussion of the monodromy for the polylogarithm; a much more abstract discussion is given in [6, 9, 13, 3]. 
Thus, this section provides a discussion; note that the correct manipulations to move from one sheet to another can be somewhat treacherous and confusing.

The inversion formula 8.1 suggests a way to move around the branch point at $z=1$. Suppose one starts at the point $z=x+i \varepsilon$ with $x$ real, positive, and greater than one, and $\varepsilon$ some arbitrarily small positive real number; thus $z$ is very near the branch cut of the principal sheet. One wishes to compare this to the straddling value at $x-i \varepsilon$. Applying the inversion formula, one can bounce these two points inside the unit circle, where there is no cut, and thus the polylog differs by $O(\varepsilon)$. The difference across the cut is thus

$\Delta=\mathrm{Li}_{s}(x+i \varepsilon)-\mathrm{Li}_{s}(x-i \varepsilon)=e^{i \pi s / 2} \frac{(2 \pi)^{s}}{\Gamma(s)}\left[\zeta\left(1-s, \frac{\ln x+i \varepsilon}{2 \pi i}\right)-\zeta\left(1-s, \frac{\ln x-i \varepsilon}{2 \pi i}\right)\right]+O(\varepsilon)$

Now, since $x>1$, a naive application of this formula yields $\Delta=0$ since $\log (x+i \varepsilon)-$ $\log (x-i \varepsilon)=O(\varepsilon)$; but clearly $\Delta$ cannot be zero. To resolve this situation, one must make the Ansatz that the cut of the logarithm should be crossed. This may be done by taking the cut of the logarithm to extend to the right, instead of to the left, as it is by convention. One then gets $\log (x+i \varepsilon)-\log (x-i \varepsilon)=2 \pi i N+O(\varepsilon)$ for some integer $N$. The difference across the cut, for positive $N$, is then

$$
\zeta(s, q)-\zeta(s, N+q)=\sum_{k=0}^{N-1} \frac{1}{(k+q)^{s}}
$$

with $q=\ln (x) / 2 \pi i$. By swinging the cut of the logarithm so that it extends to the right, one obtains that the real part of $q$ is positive; the real part of $q$ runs between 0 and 1 . As a result, the value of $(k+q)^{s}$ is unambiguous; as otherwise taking something to the power $s$ also begs the question of which sheet the power must be taken on. That is, for general complex $w$, the power function is multi-sheeted:

$$
w^{s}=e^{s \ln w} \rightarrow e^{s(\ln w+2 \pi i M)}=e^{2 \pi i s M} w^{s}
$$

for some integer $M$ representing the sheet of the power function. Since the real part of $q$ is taken as positive, one can temporarily operate with the assumption that $M=0$.

Taking $N=1$, the above reasoning provides an excellent description, which may be verified numerically. The concentric semi-circles visible in the image .5 can be entirely explained by the behavior of $q^{s}$ as $q \rightarrow 0$, that is, as $z \rightarrow 1$. Thus, the difference between the $N=1$ sheet and the $N=0$ sheet is

$$
\Delta_{1}=e^{i \pi s / 2} \frac{(2 \pi)^{s}}{\Gamma(s)}\left(\frac{\ln z}{2 \pi i}\right)^{s-1}
$$

Properly speaking, $\Delta_{1}$ is a function of $s$ and $z$, and so one should write $\Delta_{1}(s ; z)$ to signify this. However, for ease of notation, this marking is dropped, and is taken implicitly in what follows. The difference $\operatorname{Li}_{s}(z)-\Delta_{1}$ is illustrated in figure .7, the concentric rings are seen to be cancelled out precisely, leaving behind a smoothly varying function, having the expected smooth structure. Moving across the cut, for $\mathfrak{R} z>1$, the joint appears to be smooth. For general positive $N$, it is easy to confirm numerically that the discontinuity between the $N^{\prime}$ th sheet, and the $N-1$ 'th sheet, across the $\mathfrak{R} z>1$ cut is

$$
\Delta_{N}=e^{i \pi s / 2} \frac{(2 \pi)^{s}}{\Gamma(s)}\left(N-1+\frac{\ln z}{2 \pi i}\right)^{s-1}
$$


The discontinuity across the cut $\Re_{z}>1$, for negative $N$, follows similarly. For $N=-1$, the difference is given by $-1 /(-1+q)^{1-s}=e^{-i \pi s} /(1-q)^{1-s}$, or

$$
\Delta_{-1}=e^{-i \pi s / 2} \frac{(2 \pi)^{s}}{\Gamma(s)}\left(1-\frac{\ln z}{2 \pi i}\right)^{s-1}
$$

The figure.8 illustrates the difference $\operatorname{Li}_{S}(z)-\Delta_{-1}$. For general negative $N$, the difference between adjacent sheets is then

$$
\Delta_{-N}=e^{-i \pi s / 2} \frac{(2 \pi)^{s}}{\Gamma(s)}(N-q)^{s-1}
$$

Again, it can be numerically verified that that the transition from sheet to sheet is smooth as one crosses the cut $\Re_{z}>1$.

It is perhaps more clear to use explicit topological language. Let $g_{1}$ represent the homotopy class of all loops based at some point $z$ on the complex plane, that wind once around the branch-point $z=1$ in the positive direction. The action of $g_{1}$ on the polylogarithm has the effect of carrying the polylog from one sheet to the next. In the above discussion, it was determined that

$$
g_{1} \cdot \mathrm{Li}_{s}(z)=\mathrm{Li}_{s}(z)-\Delta_{1}
$$

The logarithm in $\Delta_{1}$ has a branch point at $z=0$. That is, after acting with $g_{1}$, one is now on a sheet with a cut extending from $z=0$ to the right. Let let $g_{0}$ represent the homotopy class of loops that wind once around the branch point $z=0$ in a right-handed fashion. Acting on the logarithm, one has

$$
g_{0} \cdot \ln z=\ln z+2 \pi i
$$

Recalling the definition of $q=\ln z / 2 \pi i$, one thus has $g_{0} \cdot q=q+1$, and so

$$
g_{0} \cdot \Delta_{N}=\Delta_{N+1}
$$

The principal sheet of the polylogarithm has no branch point at $z=0$, and so one has

$$
g_{0} \cdot \operatorname{Li}_{s}(z)=\operatorname{Li}_{s}(z)
$$

Similarly, the logarithm has no branch point at $z=1$, and so

$$
g_{1} \cdot \ln z=\ln z
$$

Consider now the task of pasting sheets together, such that the result is continuous as one crosses the cut $z>1$. Some confusion may result here: one must paste together the cut of the polylog, and (if any), the cut of the logarithm as well. Thus, for example, suppose one is on the $g_{1} \cdot \mathrm{Li}_{s}(z)$ sheet. To get to a sheet by means of a continuous, differentiable glueing across the $z>1$ cut, one must move as

$$
g_{1} g_{0} g_{1} \cdot \mathrm{Li}_{S}(z)=g_{1} g_{0} \cdot\left[\operatorname{Li}_{S}(z)-\Delta_{1}\right]=\operatorname{Li}_{S}(z)-\Delta_{1}-\Delta_{2}
$$

Repeating this gluing $n$ times, so that each time, one pastes the sheets so that crossing the $z>1$ cut is smooth and differentiable, gives

$$
\begin{aligned}
\left(g_{1} g_{0}\right)^{n-1} g_{1} \cdot \mathrm{Li}_{s}(z) & =\operatorname{Li}_{s}(z)-\sum_{k=1}^{n} \Delta_{k} \\
& =\operatorname{Li}_{s}(z)-e^{i \pi s / 2} \frac{(2 \pi)^{s}}{\Gamma(s)} \sum_{k=1}^{n} \frac{1}{(k-1+q)^{1-s}} \\
& =\operatorname{Li}_{s}(z)-e^{i \pi s / 2} \frac{(2 \pi)^{s}}{\Gamma(s)}\left[\zeta\left(1-s, \frac{\ln z}{2 \pi i}\right)-\zeta\left(1-s, n+\frac{\ln z}{2 \pi i}\right)\right]
\end{aligned}
$$

which is recognizable from the initial considerations. 
Winding in the opposite direction, one has

$$
g_{1}^{-1} \cdot \mathrm{Li}_{s}(z)=\mathrm{Li}_{s}(z)-\Delta_{-1}
$$

and to repeat this process, as above, one considers the action of $\left(g_{1}^{-1} g_{0}^{-1}\right)^{n} g_{1}^{-1}$ on $\mathrm{Li}_{s}(z)$.

In the development above, in the joining of sheets across $\mathfrak{R} z>1$, the cut that runs between $z=0$ and $z=1$ was mostly ignored. Visually, the cut can be clearly seen in figure .8. Consider now the task of glueing sheets across this cut. For a point $z$ just above the line connecting $z=0$ and $z=1$, one has $q=\varepsilon+i v$ for some small, positive $\varepsilon$ and some general, positive $v$. Just below this line, one has $q=1-\varepsilon+i v$. That is, $q$ differs by 1 , of course. This cut cannot be glued to the polylog, of course; it must be glued to another sheet of the logarithm. The correct gluing, for $z=x$ real, $0<x<1$, is given by

$$
\lim _{\varepsilon \rightarrow 0}\left[\Delta_{1}(x+i \varepsilon)+e^{i 2 \pi s} \Delta_{-1}(x-i \varepsilon)\right]=0
$$

That is,

$$
g_{0} \cdot \Delta_{-1}=-e^{-i 2 \pi s} \Delta_{1}
$$

This somewhat strange form is nothing more than an accounting trick; it is the result of providing a definition of $\Delta_{-N}$ that had a "natural" normalization, avoiding a minus sign. The price of that definition is this glitch. In all other respects, the homotopy proceeds as expected, so that, for example,

$$
g_{0} \cdot \Delta_{-N}=\Delta_{-N+1}
$$

for $N>1$.

The free combinations of powers of the two operators $g_{0}$ and $g_{1}$ generate a group, the monodromy group of the polylogarithm. For $s=m$ a positive integer, the monodromy group has a finite-dimensional representation of dimension $m+1$. Well-known is the case for $s=2$, the dilogarithm, where the representation forms the discrete Heisenberg group. This may be seen as follows. For $s=2$, one has

$$
\Delta_{n}=2 \pi i(\ln z+(n-1) 2 \pi i)
$$

Repeated applications of $g_{0}$ and $g_{1}$ result in a linear combinations of $\operatorname{Li}_{2}(z), \ln z$ and 1 ; no terms of any other type appear. One may take these three elements as the basis of a three-dimensional vector space. Writing the basis as a column vector,

$$
\left[\begin{array}{c}
\operatorname{Li}_{2}(z) \\
-2 \pi i \ln z \\
4 \pi^{2}
\end{array}\right]
$$

one then has the action

$$
g_{0}=\left[\begin{array}{lll}
1 & 0 & 0 \\
0 & 1 & 1 \\
0 & 0 & 1
\end{array}\right] \quad \text { and } \quad g_{1}=\left[\begin{array}{ccc}
1 & 1 & 0 \\
0 & 1 & 0 \\
0 & 0 & 1
\end{array}\right]
$$

which may be seen to be the generators of the discrete Heisenberg group $\mathcal{H}_{3}(\mathbb{Z})$. The general group element is

$$
\left[\begin{array}{lll}
1 & a & b \\
0 & 1 & c \\
0 & 0 & 1
\end{array}\right]
$$

for integers $a, b, c$. By convention, one writes $x$ for $g_{0}$ and $y$ for $g_{1}$, and defines a new element $z$ (having no relation at all to the argument of the dilogarithm) as the commutator 
$z=x y x^{-1} y^{-1}$, so that

$$
z=\left[\begin{array}{lll}
1 & 0 & 1 \\
0 & 1 & 0 \\
0 & 0 & 1
\end{array}\right]
$$

The element $z$ is in the center, so that it commutes with $x$ and $y$, so $z x=x z$ and $z y=y z$. These relations may be taken as giving the group presentation, so that

$$
\mathcal{H}_{3}(\mathbb{Z})=\left\{(x, y): z=x y x^{-1} y^{-1}, z x=x z, z y=y z\right\}
$$

For $s=3$, one may write

$$
g_{0}=\left[\begin{array}{llll}
1 & 0 & 0 & 0 \\
0 & 1 & 2 & 1 \\
0 & 0 & 1 & 1 \\
0 & 0 & 0 & 1
\end{array}\right] \quad g_{1}=\left[\begin{array}{llll}
1 & 1 & 0 & 0 \\
0 & 1 & 0 & 0 \\
0 & 0 & 1 & 0 \\
0 & 0 & 0 & 1
\end{array}\right] \text { for basis }\left[\begin{array}{c}
\operatorname{Li}_{3}(z) \\
-\pi i(\ln z)^{2} \\
2 \pi^{2} \ln z \\
4 \pi^{3} i
\end{array}\right]
$$

The generated group is no longer the Heisenberg group, as there simply aren't enough generators for that. The commutator $w=g_{0} g_{1} g_{0}^{-1} g_{1}^{-1}$ is not in the center, since $w g_{0} \neq g_{0} w$.

For general integer $s=m$, the monodromy group is always unipotent, and thus a nilpotent group. The generators take the form

$$
g_{0}=\left[\begin{array}{cccc}
1 & 0 & \cdots & 0 \\
0 & \\
\vdots & C \\
0 &
\end{array}\right] \quad g_{1}=\left[\begin{array}{ccccc}
1 & 1 & 0 & \cdots & 0 \\
0 & 1 & & \vdots \\
\vdots & & \ddots & 0 \\
0 & \cdots & 0 & 1
\end{array}\right]
$$

where $C$ are the binomial coefficients written in an upper-triangular fashion. This form is determined by taking as the basis vectors the monomials appearing in the expansion of $(\ln z+2 \pi i)^{m}$ (whence the binomialcoefficients), and normalizing with a factor of $2 \pi i / \Gamma(m)$. This discrete group does not seem to be named or well-known. Note that the existence of polylogarithm ladders seems to be equivalent to the statement that products of monodromy groups can be factored in interesting and unusual ways.

For $s$ not an integer, the action of $g_{0}$ does not close, and the general vector-space representation is infinite dimensional. The action of the $g_{0}$ monodromy can be understood to be the shift operator on an infinite-dimensional vector space, whose basis vectors may be taken to be $\Delta_{N}$; the operator $g_{1}$ acts to inject into this shift space. It is not clear whether there may also be smaller, finite-dimensional representations for particular values of $z$.

The analog of the Dirichlet $L$-functions are functions of the form

$$
p^{-s} \sum_{m=1}^{p-1} \chi(m) \operatorname{Li}_{s}\left(z e^{2 \pi i m / p}\right)
$$

where $\chi(m)$ is a primitive character modulo $p$. These functions have the strange property of having branch points at $e^{2 \pi i m / p}$ whenever $\chi(m)$ is not zero. The resulting monodromy groups have a more complex structure as well.

\section{The "Periodic" Zeta Function}

The ability to compute the polylogarithm allows one to visualize some of the interesting sums that occur in number theory. One such example is the so-called "periodic zeta 
function", defined by Apostol[2, Thm 12.6] as

$$
F(q ; s)=\sum_{n=1}^{\infty} \frac{e^{i 2 \pi n q}}{n^{s}}
$$

Clearly, one has $F(q, s)=\operatorname{Li}_{s}\left(e^{2 \pi i q}\right)$. The periodic zeta function occurs when one attempts to state a reflection formula for the Hurwitz zeta function, as

$$
\zeta(1-s, q)=\frac{\Gamma(s)}{(2 \pi)^{s}}\left[e^{-i \pi s / 2} F(q ; s)+e^{i \pi s / 2} F(1-q ; s)\right]
$$

What makes the periodic zeta function interesting is that it is not actually periodic. That one might think it is seems obvious from the definition 11.1 the direct substitution of $q \rightarrow q+1$ gives the false suggestion of periodicity in $q$. This is false because, in fact, $\operatorname{Li}_{s}(z)$ has a branch point at $z=1$. The "periodic" zeta function is multi-sheeted, and, attempting to trace a continuous path from $q \rightarrow q+1$, while keeping $q$ real carries one through the branch-point, and from one sheet to the next. This is illustrated graphically, in figure 11.1

The figure 11.1 shows an oscillatory behavior that clearly diverges as $q \rightarrow 0$. This oscillation is can be simply explained. Substituting $s=\frac{1}{2}+i \tau$ into equation 11.2 gives

$$
\zeta\left(\frac{1}{2}-i \tau, q\right)=\frac{e^{-i \pi / 4} e^{-i \tau \log 2 \pi} e^{i \phi}}{\sqrt{2 \cosh \tau \pi}}\left[e^{\pi \tau / 2} F(q ; s)+i e^{-\pi \tau / 2} F(1-q ; s)\right]
$$

after the substitution of

$$
\Gamma\left(\frac{1}{2}+i \tau\right)=\frac{\sqrt{\pi} e^{i \phi}}{\sqrt{\cosh \tau \pi}}
$$

where $\phi$ is a purely real phase that can be expressed as a somewhat complicated sum[1, eqn 6.1.27]. For reasonably large, positive $\tau$, such as $\tau=25$ in the picture, one may ignore the second term, so that the whole expression simplifies to

$$
F\left(q ; \frac{1}{2}+i \tau\right)=\exp i\left(\frac{\pi}{4}-\phi+\tau \log 2 \pi\right) \zeta\left(\frac{1}{2}-i \tau, q\right)+O\left(C^{-\tau}\right)
$$

for some real constant $C>0$. Then, as $q \rightarrow 0$, the leading contribution to the Hurwitz zeta comes from the $n=0$ term in equation 1.1] that is, $q^{-s}=e^{i \tau \log q} / \sqrt{q}$, so that

$$
F\left(q ; \frac{1}{2}+i \tau\right) \approx \frac{e^{i \tau \log q} e^{i \psi}}{\sqrt{q}} \text { as } q \rightarrow 0
$$

for some fixed, real phase $\psi$ that is independent of $q$. As is eminently clear from both the picture 11.1, and from the approximation 11.3, the limit of $q \rightarrow 0$ of $F(q, s)$ cannot be taken: this is the branch-point of $\operatorname{Li}_{s}(z)$ at $z=1$.

Curiously, the limit $q \rightarrow 1$ does exist, and one has $F(1, s)=\zeta(s)$ the Riemann zeta. The approximation 11.3 hints at how to move through the branch point, from one sheet to the next: 11.3 is the discontinuity between sheets. That is, one has, for large, positive $\tau$

$$
F\left(q+1 ; \frac{1}{2}+i \tau\right) \approx F\left(q, \frac{1}{2}+i \tau\right)-\frac{e^{i \tau \log q} e^{i \psi}}{\sqrt{q}}
$$

as the formula that approximates the movement from one sheet to the next. In essence, this shows that the "periodic" zeta function is not at all periodic: $F(q+1 ; s) \neq F(q ; s)$ whenever $\Re s \leq 1$.

The complete relationship between the Hurwitz zeta and the periodic zeta is rather subtle. For example, if instead one considers large negative $\tau$, and graphs the periodic zeta, 
FIGURE 11.1. Periodic zeta function

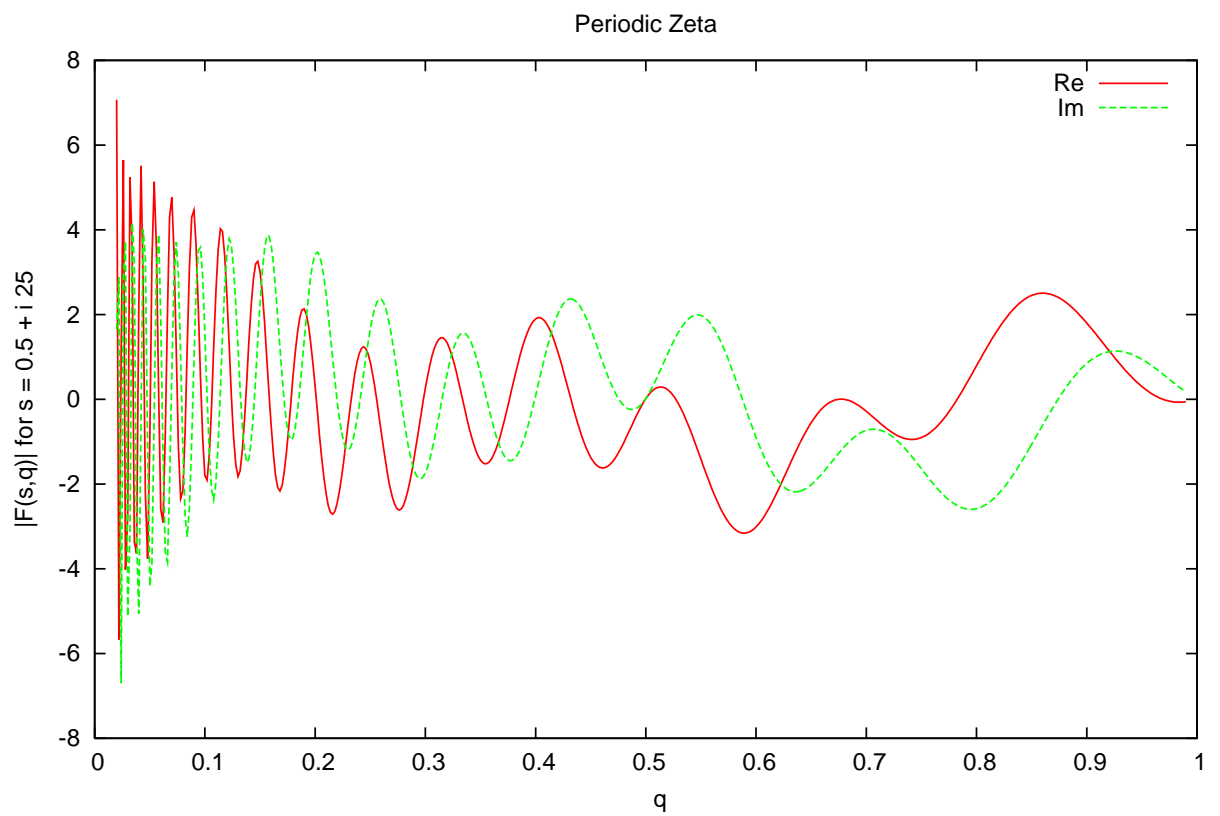

This graph shows the real and imaginary parts of the periodic zeta function

$$
F(q, s)=\operatorname{Li}_{s}\left(e^{2 \pi i q}\right)
$$

for $s=\frac{1}{2}+i 25$. This value of $s$ is close to a zero of the Riemann zeta function, at $s=\frac{1}{2}+i 25.01085758014 \ldots$ Thus, both the real and imaginary parts approach zero at $q=1$, as well as at $q=\frac{1}{2}$. The increasing oscillations as $q \rightarrow 0$ are due to the contribution of the very first term of the Hurwitz zeta: that is, these oscillations are nothing other than that of $q^{-s}=e^{i 25 \log q} / \sqrt{q}$. Subtracting these away is the same as analytically continuing

to the region $q>1$, and matches the coarser set of oscillations, which are given by

$$
(1+q)^{-s}=e^{i 25 \log (1+q)} / \sqrt{1+q}
$$

Noteworthy is that the presumed "periodicity" in $q$ is very misleading: the image suggests an essential singularity at $q=0$, and continuing, logarithmically slower oscillatory behavior for the analytic continuation to the region where $q>1$.

one obtains what is essentially the left-right reversed image of 11.1, with oscillations approaching a singularity at $q \rightarrow 1$. One may easily numerically verify that these oscillations are precisely of the form $(1-q)^{-s}$. From this, one may deduce that the correct form of the reflection formula is

$$
F(q, 1-s)=\frac{\Gamma(s)}{(2 \pi)^{s}}\left[e^{i \pi s / 2} \zeta(s, q)+e^{-i \pi s / 2} \zeta(s, 1-q)\right]
$$

which captures the oscillatory behavior at $q \rightarrow 0$ for large positive $\tau$ and the oscillations at $q \rightarrow 1$ for large negative $\tau$.

The constraint of keeping $q$ real causes integer values of $q$ to correspond precisely to the branch point of the polylogarithm. This makes reasoning about the continuity of the periodic zeta at integral values of $q$ rather turbid. Since the Riemann zeta function lies 
precisely at the branch point, this seems to also make it difficult to gain new insight into the Riemann zeta in this way.

\section{CONCLUSION}

The updated algorithm appears to offer a stable and fast way of computing the Hurwitz zeta function for general complex $s$ and real $q$, and the polylogarithm for general complex $s$ and $z$. An actual implementation using a variable-precision library shows that the algorithm is quite tractable.

An unexplored area is the optimal implementation of the algorithm using standard IEEE double-precision floating-point mathematics. Preliminary work shows that some of the intermediate terms are just large enough so that rounding error may be of concern; the region of high-precision convergence has not been characterized. The propagation of rounding errors through the algorithm has not been characterized; it may be possible to re-arrange terms to minimize the propagation of errors. Such a characterization is necessary before reliable double-precision code can be created. By contrast, such considerations can be swept under the rug when employing arbitrary-precision arithmetic.

Evaluating the bounds 6.3 and 6.4 so as to obtain an order estimate, is non-trivial, and can account for a significant amount of compute time. A speedier heuristic is desirable.

It may be possible to find even more rapidly converging algorithms, by generalizing some of the results from Cohen et al[17], or by appealing to the general theory of Padé approximants. Both approaches seem promising, although that of Padé approximants may yield a more general theory.

The polylogarithm and Hurwitz zeta functions are both special cases of the Lerch transcendental

$$
\sum_{n=0}^{\infty} \frac{z^{n}}{(n+q)^{s}}
$$

It should be possible to extend the techniques in this paper to provide a rapid, globally convergent algorithm for the Lerch transcendental, thus enabling a deeper numerical exploration of its peculiarities.

A further exploration of the monodromy group of the polylogarithm seems to be merited; although this does not appear to be terribly challenging, it would be nice to have the details at hand.

This paper concludes with the application of the algorithm to render some pretty images.

\section{REFERENCES}

1. Milton Abramowitz and Irene A. Stegun (eds.), Handbook of mathematical functions, 10th printing ed., Dover Publications, 1972.

2. Tom M. Apostol, Introduction to analytic number theory, Springer; New York, 1976.

3. Spencer Bloch, Structural properties of polylogarithms, Mathematical Surveys and Monographs, vol. 37, ch. "Introduction to Higher Logarithms", pp. 275-285, American Mathematical Society, 1991.

4. P. Borwein, An efficient algorithm for the riemann zeta function, http://www.cecm.sfu.ca/personal/pborwein/PAPERS/P117.ps, January 1995.

5. _ An efficient algorithm for the riemann zeta function, Constructive experimental and nonlinear analysis, CMS Conference Proceedings 27, 2000, pp. 29-34.

6. Ovidiu Costin and Stavros Geroufalidis, Resurgence of the fractional polylogarithms, arXiv math.CA/0701743 (2007).

7. R. E. Crandall, Note on fast polylogarithm computation, www.reed.edu/ crandall/papers/Polylog.pdf, January 2006.

8. Free Software Foundation, Gnu multiple precision arithmetic library, http://www.swox.com/gmp/.

9. Richard M. Hain, Classical polylogarithms, arXiv alg-geom/9202022 (1992). 
10. Helmut Hasse, Ein summierungsverfahren fur die riemannsche ?-reihe, Mathematische Zeitschrift 32 (1930), 458-464.

11. A. Jonquière, Notes sur la série (polylogarithm), Bull. Soc. Math. France 17 (1889), 142-152.

12. Leonard Lewin, Polylogarithms and associated functions, North Holland, 1981.

13. Richard M.Hain \& Robert Macpherson, Structural properties of polylogarithms, Mathematical Surveys and Monographs, vol. 37, ch. "Introduction to Higher Logarithms", pp. 337-353, American Mathematical Society, 1991.

14. Bateman Manuscript Project, Higher transcendental functions, vol. 1, McGraw Hill, New York, 1953.

15. Rasa Slezeviciene, An efficient algorithm for computing dirichlet l-functions, Integral Transforms and Special Functions 15 (2004), no. 6, 513-522.

16. Linas Vepstas, The bernoulli map, http://www.linas.org/math/bernoulli.pdf, 2004.

17. Henri Cohen, Fernando Rodriguez Villegas, and Don Zagier, Convergence acceleration of alternating series, Experimental Mathematics 9 (2000), no. 1, 3-12, http://www.math.utexas.edu/ villegas/publications/convaccel.pdf.

18. William T. Vetterling William H. Press, Saul A. Teukolsky and Brian P. Flannery, Numerical recipes in c, the art of scientific computing, 2nd edition ed., Cambidge University Press, 1988.

19. David Wood, The computation of polylogarithms, Tech. Report 15-92*, University of Kent, Computing Laboratory, University of Kent, Canterbury, UK, June 1992, http://www.cs.kent.ac.uk/pubs/1992/110.

\section{ApPendix: Some Pretty PiCtures}

Below follow some interesting pictures of the polylogarithm and the Hurwitz zeta function for values of $s$ on the critical line $s=1 / 2+i \tau$. They are shown here, as they are not commonly known. Each figure represents hours of computation on modern computers. 
FIGURE .1. The Periodic Zeta

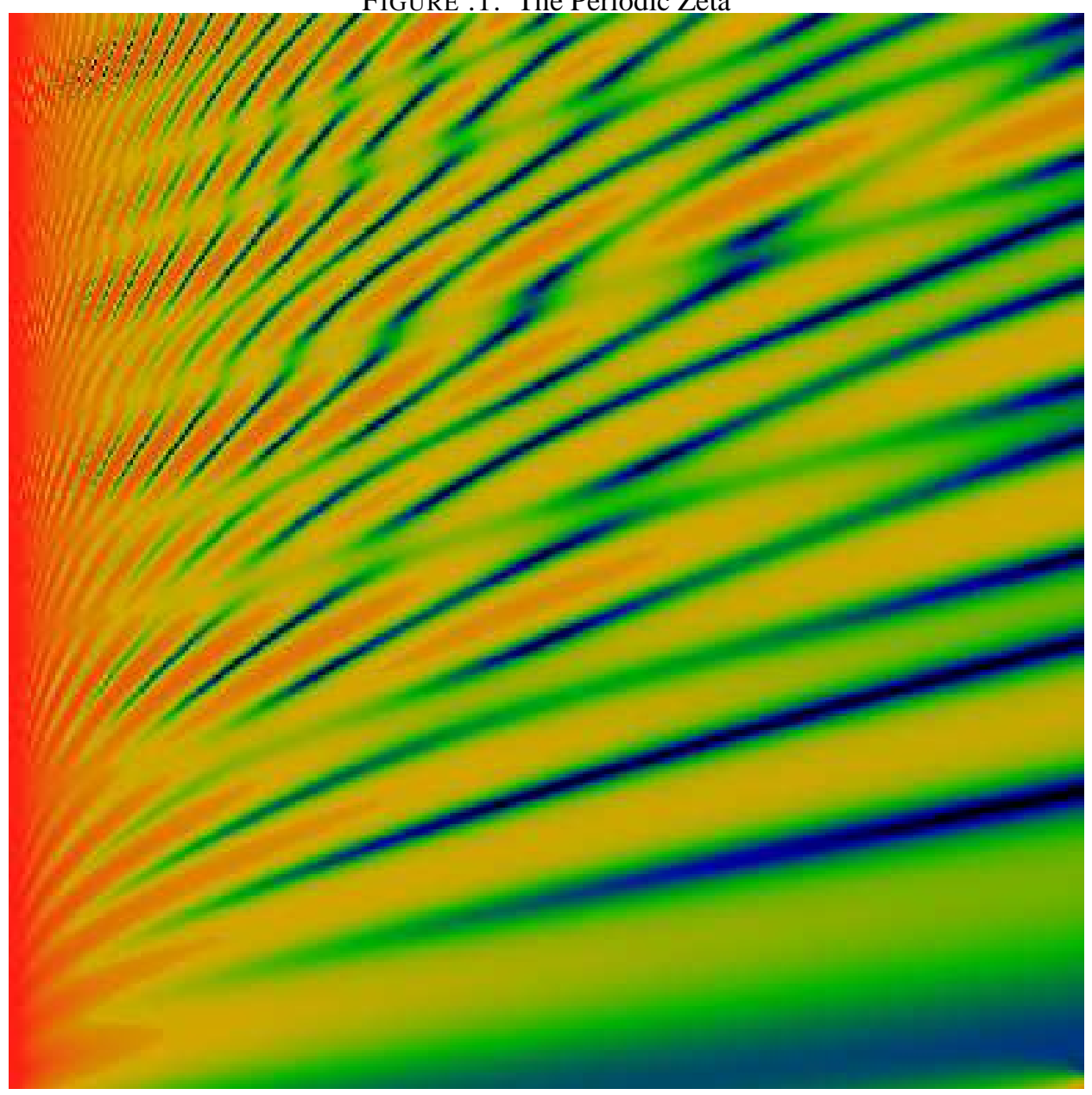

This image illustrates the so-called "periodic zeta function"

$$
F(q ; s)=\operatorname{Li}_{s}\left(e^{2 \pi i q}\right)=\sum_{n=1}^{\infty} \frac{\exp (2 i \pi n q)}{n^{s}}
$$

Graphed is the magnitude of $F(q ; s)$, with a color scale such that black represents zero, greenish-blue being values on the order of one, yellow on the order of two, with increasingly large values shown as orange-red. Along the horizontal axis runs $q$, taken to be real, from $q=0$ on the left to $q=1$ on the right. Then, writing $s=\frac{1}{2}+i \tau$, the value of $\tau$ is varied along the vertical axis, running from $\tau=0$ at the bottom of the image, to $\tau=50$ at the top of the image. Zeroes of the Riemann zeta function $\zeta(s)=F(1, s)$ are located where the blue lines intersect the right edge of the image. From the bottom, the first three zeroes are at $s=0.5+i 14.13,0.5+i 20.02,0.5+i 25.01$. Due to the relation to the Dirichlet eta function, the zeros also materialize at the same values of $s$, but on the

$$
q=1 / 2 \text { line. }
$$

Remarkably, the blue streaks seem to be roughly parabolic, but are interrupted by nearly straight "erasures". The pattern is reminiscent of graphs of the strong-field Stark effect (need ref). In the Stark effect, eigenvalues are given by the characteristic values of the Mathieu functions. These cross over one angther in a curious fashion; see for example, figure 20.1 in Abramowitz \& Stegun.

The precise form of the parabolic blue streaks are due to a term of the form $q^{-s}$, as given by equation 11.3 These may be subtracted, as illustrated in the next image. 
FIGURE .2. Hurwitz zeta, with leading term subtracted

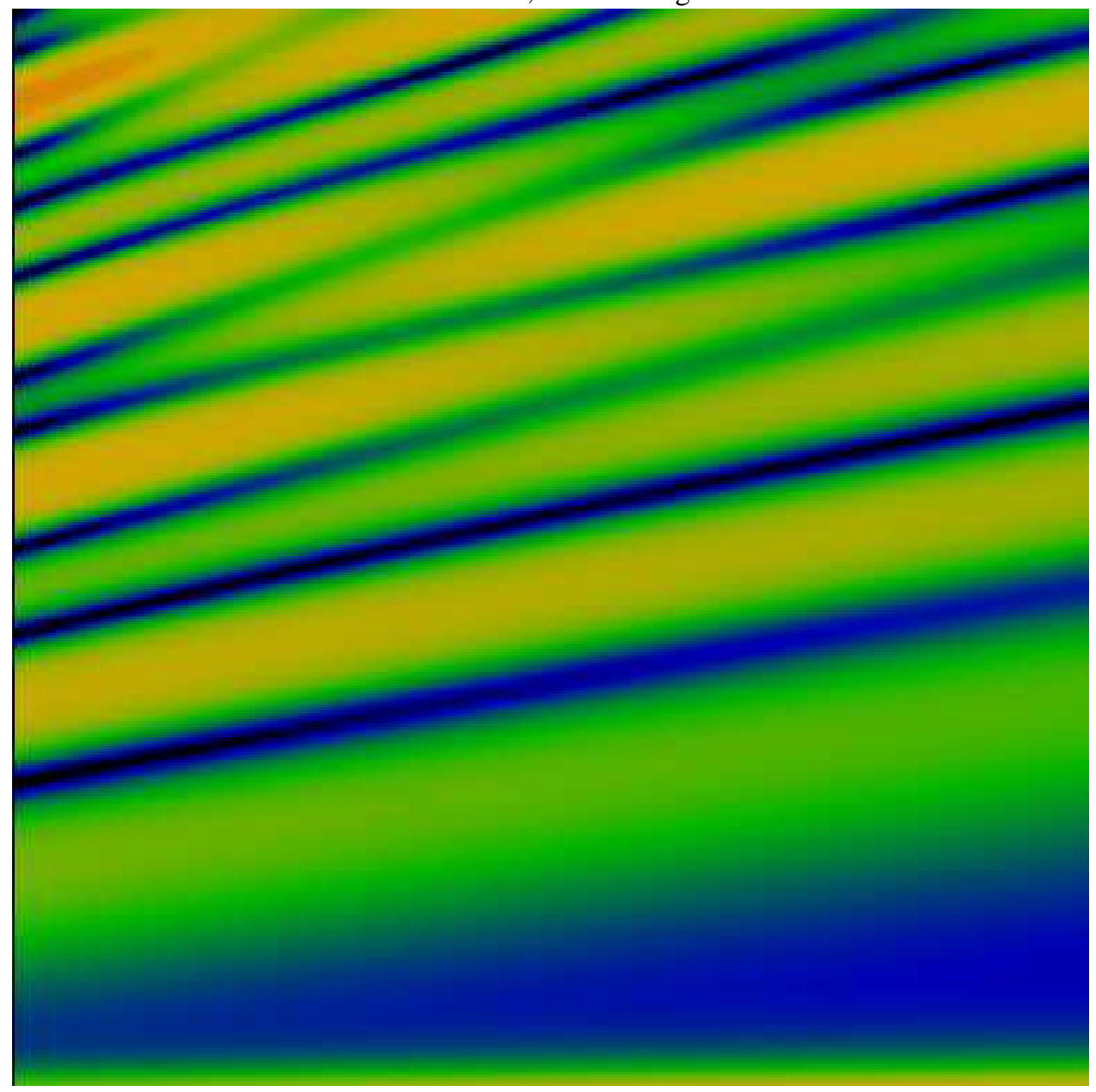

This image shows the magnitude of

$$
\zeta(s, q+1)=\zeta(s, q)-q^{-s}
$$

for the range of $0 \leq q \leq 1$ along the horizontal axis, and $0 \leq \tau \leq 50$ along the vertical axis, just as in the previous image; the same coloration scheme is used as well. This essentially corresponds to the previous picture, with the leading divergence subtracted; for large positive $\tau$, the magnitude of the periodic zeta and the Hurwitz zeta differ by an exponentially small amount. A careful comparison of this picture to the previous now shows that the "erasures" or "streaks" in the previous image correspond exactly to the dark parts of this image. Again, a criss-cross lattice pattern can be seen. The dominant streaks in this picture are presumably due to the $(1+q)^{-s}$ term. 
FIGURE .3. Extended view of Hurwitz zeta

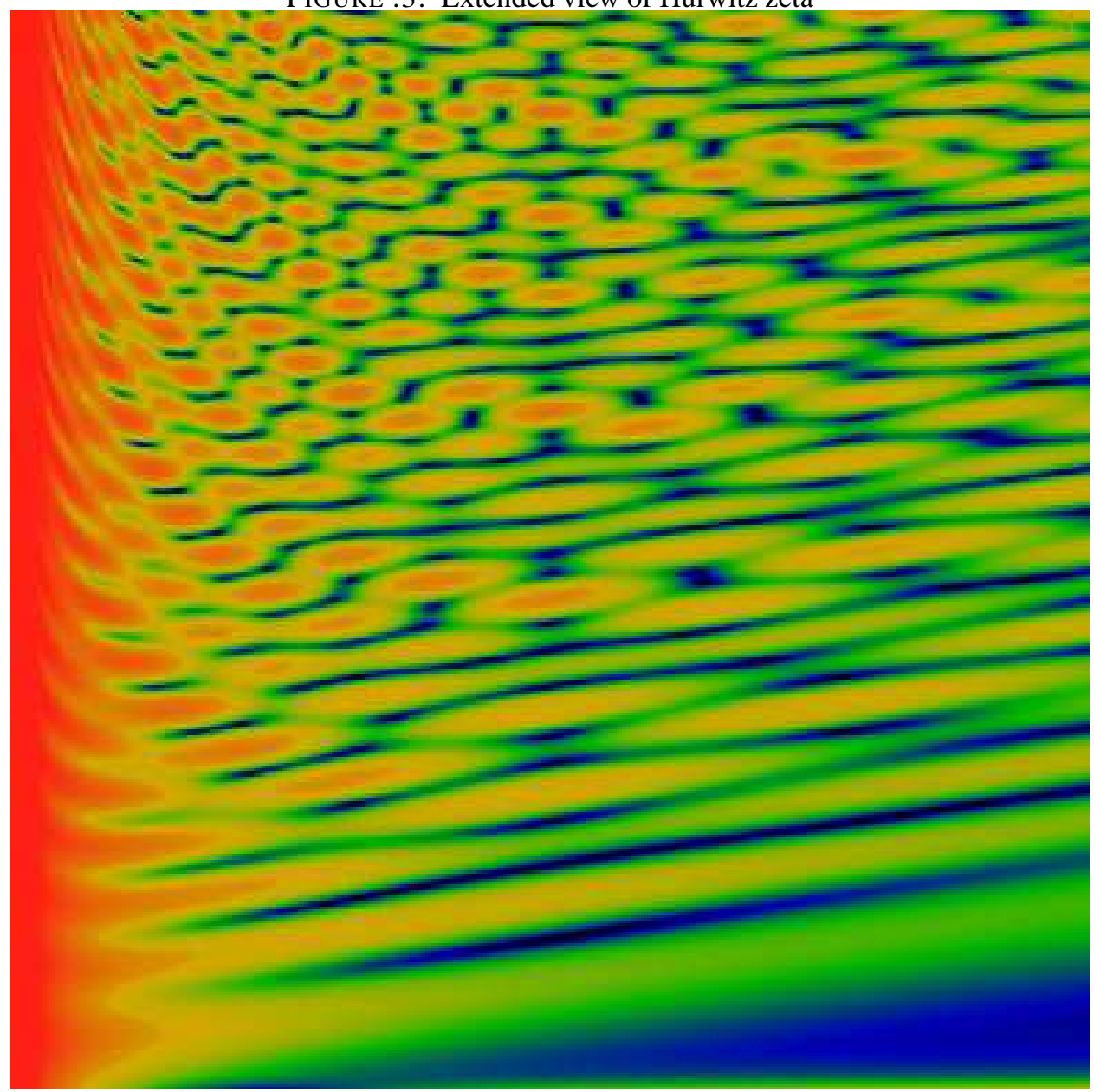

This figure shows a view of the magnitude of the Hurwitz zeta $|\zeta(s, q)|$ over an extended range, together with a rescaling of coordinates in an attempt to straighten out the striations. The value of $q$ ranges from 0 to $2 \sqrt{2} \approx 2.8$ along the horizontal axis, although it is scaled as $q^{3 / 2}$ along this axis. This rescaling essentially turns the parabolic striations into straight, radial lines emanating from the origin at the lower left. This image also re-scales the $\tau$ coordinate, in an attempt to turn the radial striations into horizontals.

The value of $q=1$ is a vertical line running exactly down the middle of the image; the non-trivial Riemann zeroes are visibly stacked up on this line. The value of $\tau$ runs from 0 to 100 on this vertical line. On other verticals, $\tau$ has been rescaled by $q^{1 / 2}$, so that $\tau$ runs from 0 to 141 on the right hand side of the image, while being essentially held at zero along the left edge of the image.

The increasing complexity of the chain-link pattern is apparent as one moves up the imaginary axis. Equally apparent is that the maximum range of excursion of the magnitude changes only slowly over the range of the image: as the pattern increases in complexity, it does not wash out, but has a distinct binary on/off character. 
FIGURE .4. Polylogarithm phase plot

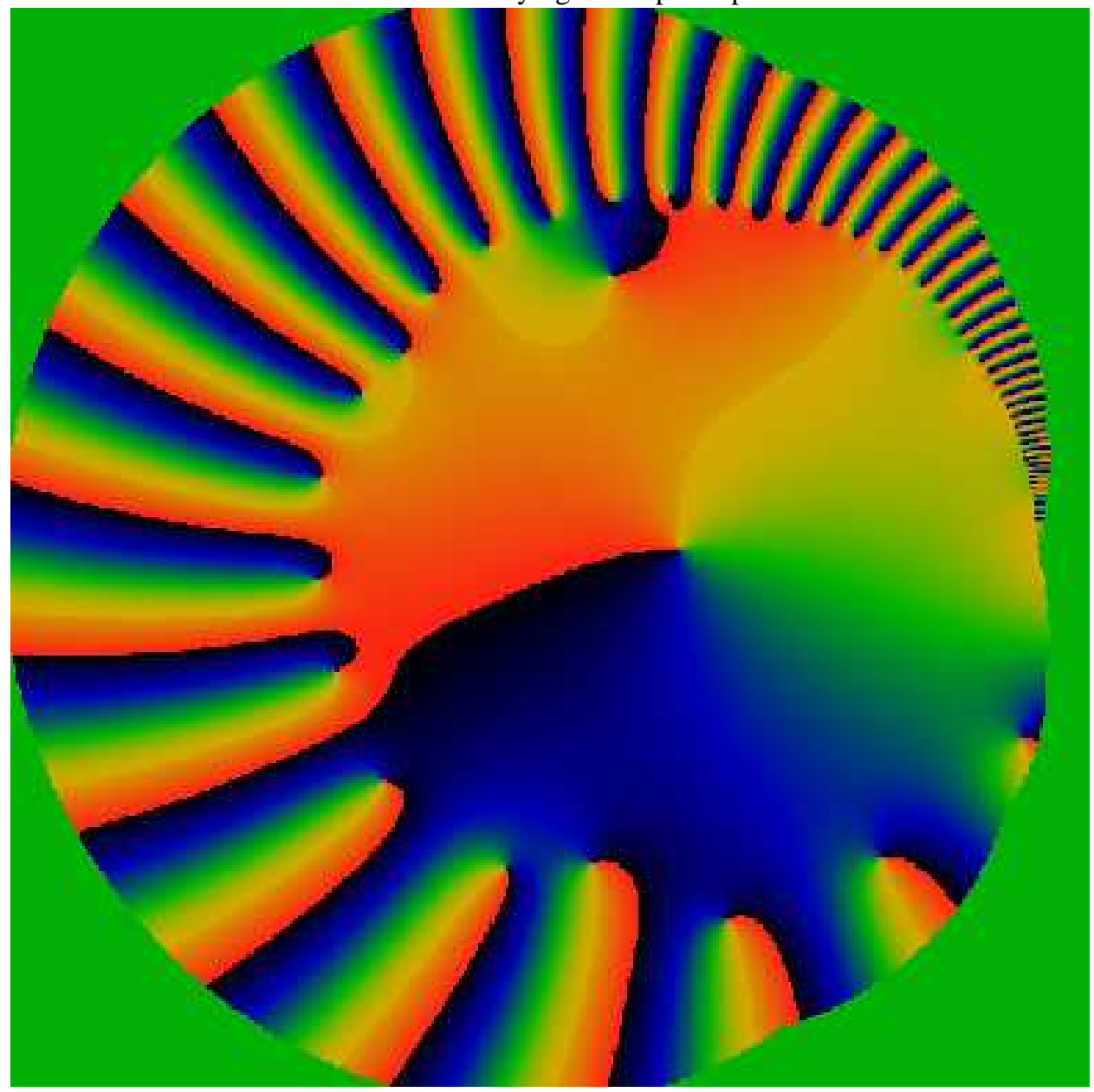

This image shows a phase plot for the polylogarithm, on the complex $z$-plane, for $s=0.5+i 80$. The color scheme is such that black represents the phase $\arg \operatorname{Li}_{s}(z)=-\pi$, red represents the value of $+\pi$, and green a phase of zero. Points where the phase wraps around the full color range are zeros. Thus, each of the "fingers" in this image terminates on a zero. Most of these zeros are close to, but usually not quite on the circle $|z|=1$. The lone zero near the center of the image corresponds to the zero at $z=0$. The slight cusp at

the right side of the image corresponds to $z=1$. The outer boundary of the image, adjoining to the solid green area, represents the limit of convergence for the combination of the Borwein algorithm and the duplication formula; one can enlarge the region of convergence slightly, but not much, but using polynomial approximations of higher order.

The boundary is easily crossed by applying the inversion formula, as the later images show. The image is offset somewhat, rather than being centered on $z=0$, because most of the convergent region is to the left of the imaginary axis.

Note the other lone zero floating along inside of the $|z|<1$ disk. If this picture were animated as $\tau$ increased from 0 in the positive direction, then the zero fingers can be see be seen as marching across in a counter-clockwise fashion, starting at $z=1$ and proceeding around. Most zeros pass to another sheet upon crossing $z=1$, after making a full revolution; others spiral around a secone time on this sheet, such as the lone zero up top. An animated movie of this image, showing the spiraling, can be seen at http://www.linas.org/art-gallery/polylog/polylog.html 
FIGURE .5. Polylog whole plane

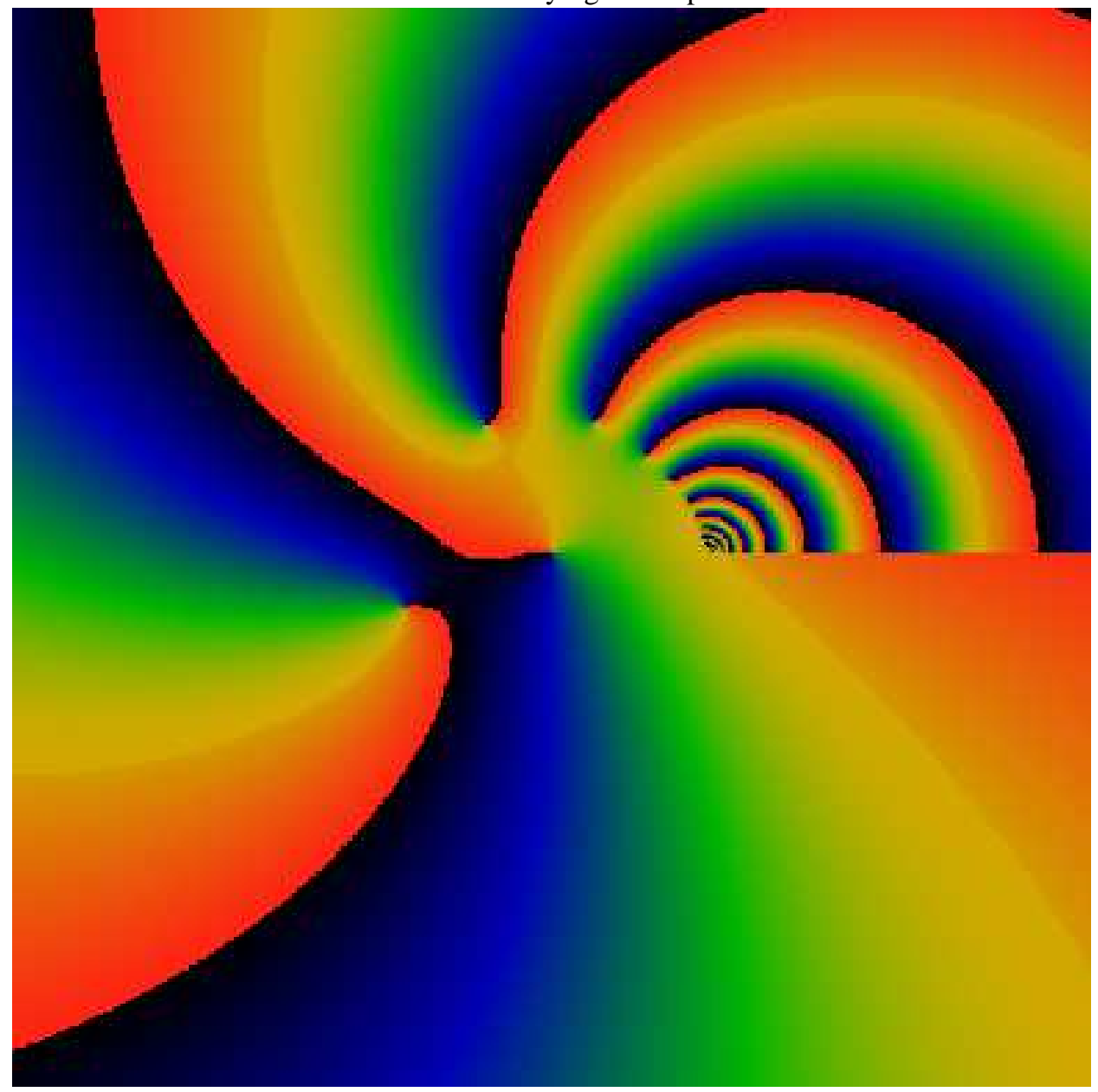

This image illustrates the results of combining the Borwein algorithm together with the inversion and duplication formulas. The image shows the complex $z$-plane, is centered on $z=0$, and runs from -3.5 to +3.5 , left to right, top to bottom. The value of $s$ is fixed at $s=0.5+15 i$ for the whole picture. The color scheme is the same as for the previous picture.

Besides the zero at $z=0$ at the center of the picture, another zero is visible to the left, and slightly down. This zero is an "ex-Riemann zero", in that, if instead one had created the image for $s=0.5+i 14.1347 \ldots$, then this point would have been located exactly at $z=-1$. As it happens, this point has now rotated to slightly below $z=-1$. The zeroes above and to the right of the origin are Riemann zeroes to be: as $\tau$ increases, each will in turn rotate counterclockwise to assume the position at $z=-1$.

The concentric shells are centered on the point $z=1$. The branch cut can be seen extending to the right from from $z=1$, on the positive real axis. 
FIGURE .6. Polylogarithm off the critical axis

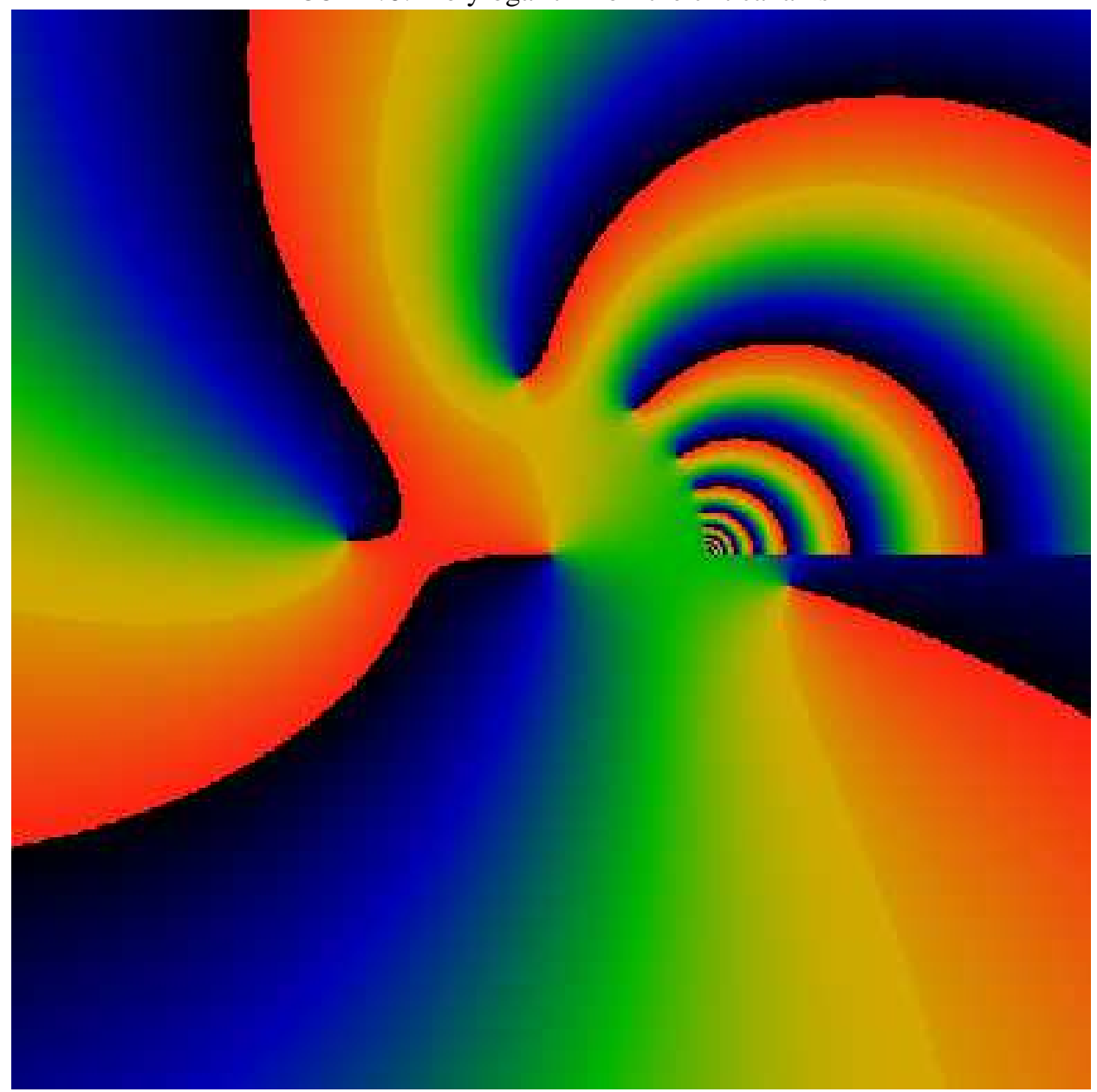

This figure shows the polylogarithm in the complex $z$-plane, at fixed $s=\sigma+i \tau=1.2+i 14$. It is superficially similar to the previous image, with two notable differences. Visible just under the cut, on the right, is a zero. If $\sigma$ were smaller, this zero would move to the left; at $\sigma=0.5$, it would be very near to the branch point at $z=1$. As $\tau$ gets larger, this point moves up, crossing the branch cut and moving onto the next sheet.

Of course, if instead one had $\sigma+i \tau=0.5+i 14.13 \ldots$, this zero would be located precisely at $z=1$. Thus, this figure illustrates what a Riemann zero looks like when it is not on the critical strip.

As $\tau$ increases, the remaining zeroes circle around in a widening spiral: thus, the zero at the far left of the image is not near $z=-1$, but is to the left of there (and thus is not going to be a zero of the Dirichlet eta function). If instead one had $\sigma$ with a value of less than 0.5 , the zeroes would spiral around in an ever-tightening spiral, and would never hit the branch cut or cross over. The Riemann hypothesis is essentially that these zeroes are impacting exactly at the branch point; a violation of RH would be a pair of zeroes that failed to hit the branch point, instead passing to the left and right of the branch point. This figure suggests that RH holds: the zeroes are clearly lined up in single file; it is hard to imagine how this single file might break ranks into a pair of zeros that simultaneously passed near the branch point. 


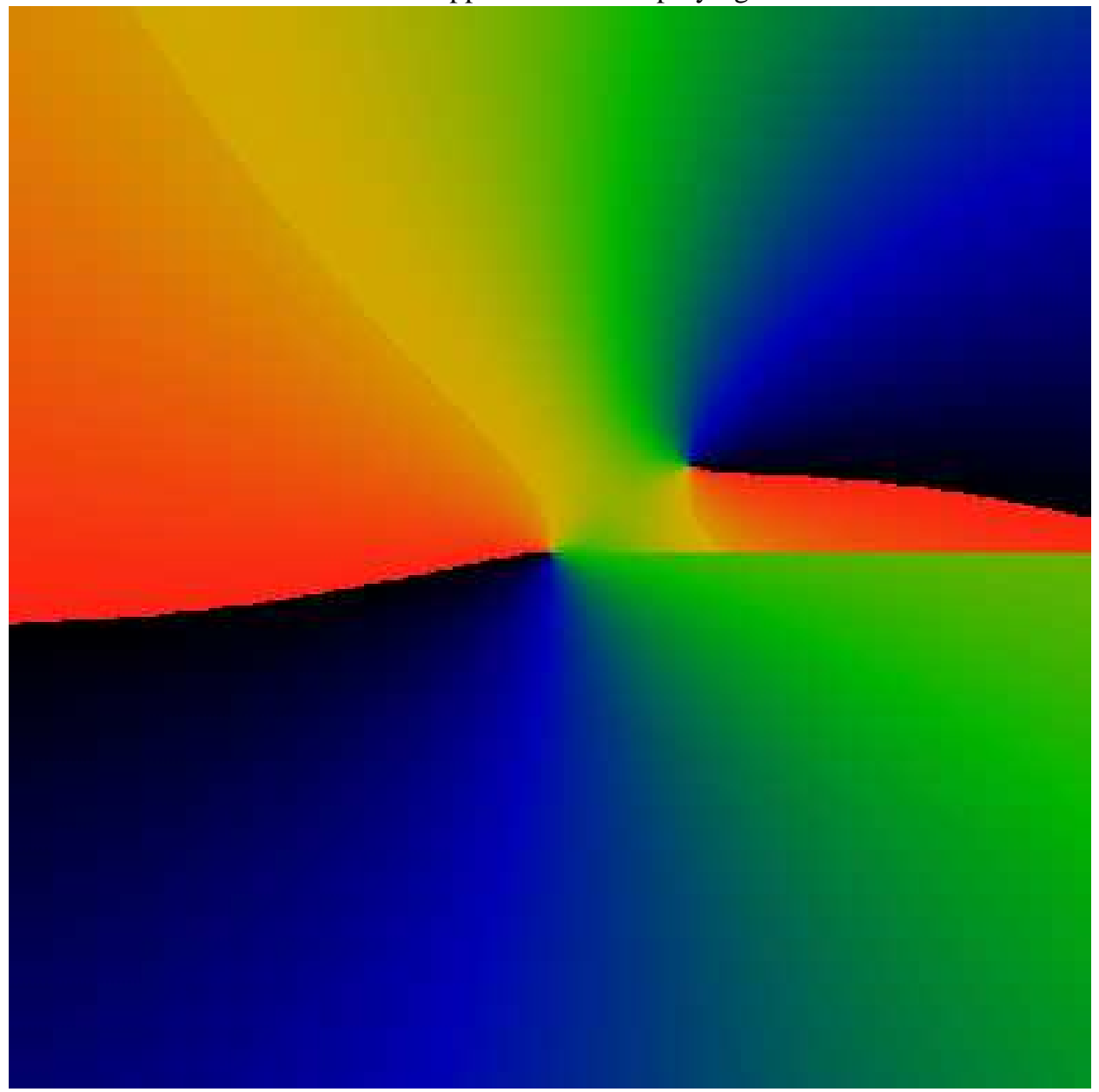

This figure shows the $g_{1} \mathrm{Li}_{s}(z)=\mathrm{Li}_{s}(z)-\Delta_{1}$ sheet of the polylogarithm on the complex $z$-plane, obtained by circling the branch-point at $z=1$ in the right-handed direction. Two zeroes are visible: one at $z=0$, and the other an "ex-Riemann zero", located not far from $z=1$, a bit above the real axis. For this image, $s$ is held constant, at $s=0.5+i 15$; the Riemann zeta function has its first non-trivial zero at $s=0.5+i 14.13 \ldots$ At that value, the above zero would have been located precisely at $z=1$, on the branch point of the polylogarithm. As $\tau$ increases, the Riemann zeroes pass precisely through this branch point, leaving the first sheet and moving to this one.

Indeed, the Riemann hypothesis can be taken as being equivalent to the statement that the Riemann zeroes always pass through the branch point in moving from one sheet to another. Taking $s=\sigma+i \tau$, if $\sigma$ is held constant at $\sigma<1 / 2$ while $\tau$ is increased, the polylogarithm zeroes never pass through the branch cut, and stay on the main sheet; whereas for $\sigma>1 / 2$, they do pass through the branch cut, but not at the branch point. 


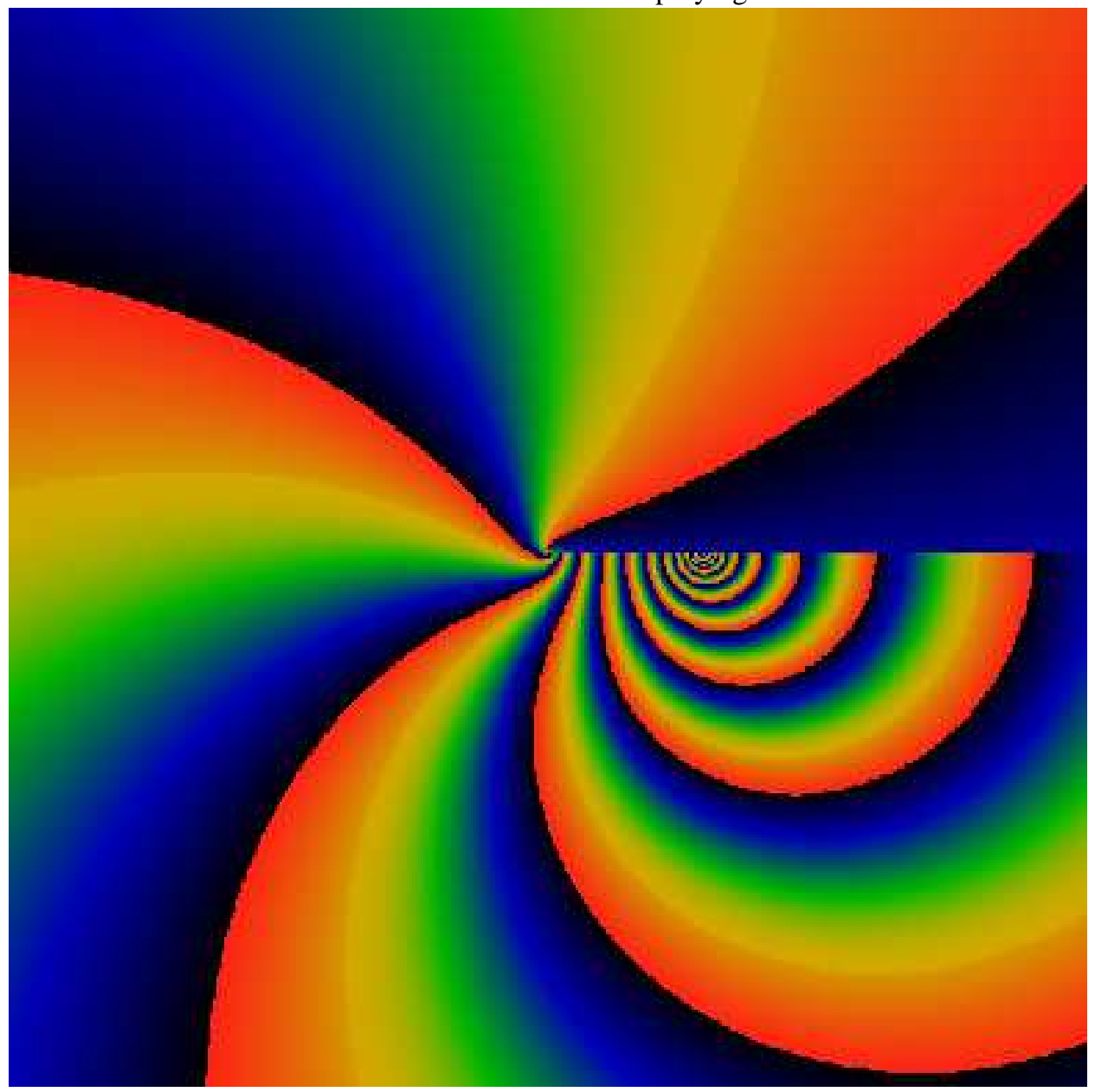

This figure shows the $g_{1}^{-1} \mathrm{Li}_{s}(z)=\mathrm{Li}_{s}(z)-\Delta_{-1}$ sheet of the polylogarithm on the complex $z$-plane, obtained by going around the branch-point at $z=1$ in the left-handed (clockwise) direction. The concentric curves are centered on $z=1$, and whose form is essentially that of $(1-q)^{1-s}$. The vertex of the black triangle approaches $z=0$. By visual inspection, it is clear how to glue this sheet to the principal sheet shown in figure.5 After this gluing, a cut remains between the points $z=0$ and $z=1$. This cut may be glued to the sheet that results by winding around the branch at $z=0$ in a clockwise manner. The result is shown in the next figure. 
FIGURE .9. More polylogarithm sheets

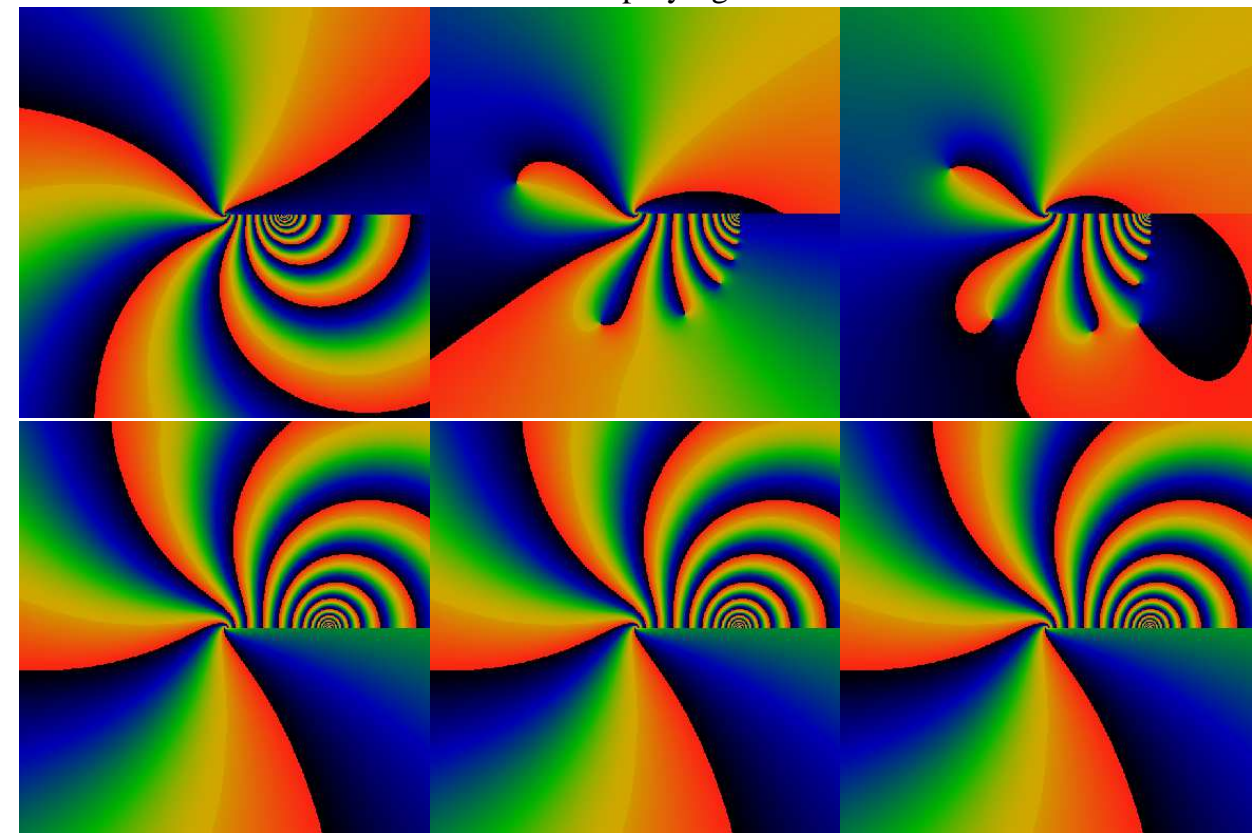

Top row: shows the result of winding around $z=1$, one, two and three times in a left-handed fashion. The cuts to the right of $z=1$ join together smoothly from image to image. Using the monodromy group notation, these are the $g_{1}^{-1}$, the $g_{1}^{-1} g_{0}^{-1} g_{1}^{-1}$, and the $g_{1}^{-1} g_{0}^{-1} g_{1}^{-1} g_{0}^{-1} g_{1}^{-1}$ sheets.

Second row: Result of winding left-handed around $z=1$, followed by winding around $z=0$ once. The cut $0<z<1$ joins smoothly to the images in the row above. The three images appear to be visually indistinguishable; but in fact they are not the same. Using

the monodromy group notation, these are the $g_{0} g_{1}^{-1}$, the $g_{0} g_{1}^{-1} g_{0}^{-1} g_{1}^{-1}$, and the $g_{0} g_{1}^{-1} g_{0}^{-1} g_{1}^{-1} g_{0}^{-1} g_{1}^{-1}$ sheets. 